\title{
Measuring Nano- to Microstructures from Relayed Dynamic Nuclear Polarization NMR
}

\author{
Arthur C. Pinon, ${ }^{\dagger}$ Judith Schlagnitweit, ${ }^{\ddagger}\|\|$ Pierrick Berruyer, $\|$ Aaron J. Rossini, ${ }^{\dagger, \perp}$ Moreno Lelli, ${ }^{\#}$ \\ Etienne Socie, ${ }^{\dagger}$ Mingxue Tang, ${ }^{{ }^{\odot}}$ Tran Pham, ${ }^{\S}$ Anne Lesage, ${ }^{\|}$Staffan Schantz, ${ }^{\bigcirc}$ and Lyndon Emsley ${ }^{*},{ }^{\dagger}$ \\ ${ }^{\dagger}$ Institut des Sciences et Ingénierie Chimiques, Ecole Polytechnique Fédérale de Lausanne (EPFL), CH-1015 Lausanne, Switzerland \\ ${ }^{\ddagger}$ Karolinska Institutet, Stockholm, Sweden \\ ${ }^{\S}$ GSK Medicines Research Centre, Stevenage SG1-2NY, United Kingdom \\ "Centre de Résonance Magnétique Nucléaire (RMN) à Très Hauts Champs, Institut des Sciences Analytiques (CNRS/ENS de \\ Lyon/UCB Lyon 1), Université de Lyon, 69100 Villeurbanne, France \\ ${ }^{\perp}$ Department of Chemistry, Iowa State University, Ames, Iowa 50011-3111, United States \\ \# Department of Chemistry, University of Florence, Center for Magnetic Resonance, 50019 Sesto Fiorentino (FI), Italy \\ ${ }^{\nabla}$ National High Magnetic Field Laboratory, 1800 East Paul Dirac Drive, Tallahassee, Florida 32310, United States \\ OPharmaceutical Development, AstraZeneca R\&D Mölndal, SE-431 83 Mölndal, Sweden
}

\section{Supporting Information}

ABSTRACT: We show how dynamic nuclear polarization (DNP) NMR can be used in combination with models for polarization dynamics to determine the domain sizes in complex materials. By selectively doping a source component with radicals and leaving the target undoped, we can measure experimental polarization buildup curves which can be compared with simulations based on heterogeneous distributions of polarization within the sample. The variation of the integrated DNP enhancement as a function of the polarization time is found to be characteristic of the geometry. We demonstrate the method experimentally on four different systems where we successfully determine domain sizes between 200 and $20000 \mathrm{~nm}$, specifically

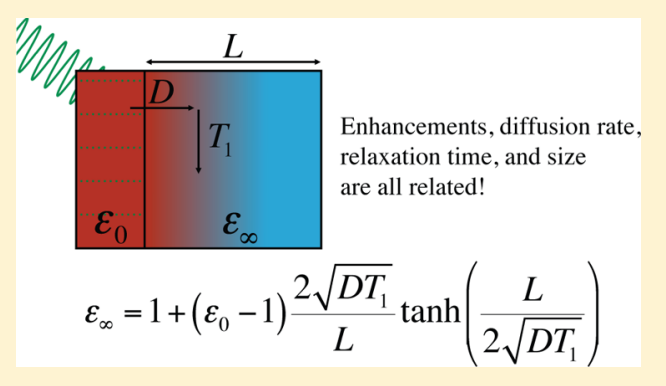
in powdered histidine hydrochloride monohydrate, pore lengths of mesoporous silica materials, and two domain sizes in twocomponent polymer film coatings. Additionally, we find that even in the apparently homogeneous frozen solutions used as polarization sources in most DNP experiments, polarization is relayed from protons near the radicals to the bulk of the solution by spin diffusion, which explains the experimentally observed buildup times in these samples.

\section{INTRODUCTION}

Modern materials such as polymers or pharmaceuticals, as well as porous materials, are usually well-designed nano- or microstructured (multicomponent) systems. The physical properties of these materials are strongly related to particle sizes, pore sizes or pore lengths, and domain sizes. Therefore, methods to determine architectures on these nano- to micrometer length scales are necessary for the development of next-generation materials.

Depending on the nature of the sample, domain sizes may be measurable by laser diffraction, scattering methods, or by electron microscopy methods. ${ }^{1-3}$ However, in complex multicomponent mixtures, these methods become much more difficult to apply because they often cannot resolve more than two components. NMR would be a method of choice in these cases. Indeed, based on chemical shift differences or relaxation properties of different compounds, NMR often allows clear distinction among several components. Consequently, the study of in situ domain sizes becomes feasible with NMR methods. 4
Proton spin diffusion experiments are the most widely used methods for domain size measurement. ${ }^{6,7}$ In these experiments, an initial spatially heterogeneous nonequilibrium distribution of magnetization is created, and the return to equilibrium driven by spin diffusion is monitored. ${ }^{8}$ In order to create the nonequilibrium distribution by selecting proton magnetization from particular domains, different procedures have been proposed including filters based on dipolar couplings, $7,9,10$ differences in relaxation properties, ${ }^{8}$ or proton or carbon chemical shift differences. ${ }^{11-15}$ These methods work well in two-component systems where the components exhibit significant differences in the properties chosen for selection. However, for more complex multiple component systems these methods are usually not feasible.

It has recently been shown that domain sizes can be determined in complex systems using dynamic nuclear

Received: May 9, 2017

Revised: June 20, 2017

Published: June 21, 2017 
polarization (DNP) where the nonequilibrium distribution of magnetization is obtained by locally enhancing polarization. ${ }^{16-19}$ It has also been shown that the selection process can be replaced by selective doping of one of the domains of the diamagnetic system with paramagnetic species and using paramagnetic relaxation enhancement to estimate the domain sizes. $^{20}$

In these approaches, the initial out-of-equilibrium state is achieved by selective doping followed by either comparison with spin diffusion dynamics in an undoped sample or comparison to a state in which the doped region is hyperpolarized. The curves obtained through comparison of the two initial states for different recovery times in saturationrecovery experiments can then be interpreted using numerical solutions of the diffusion equations.

The DNP method has previously been used to determine domain sizes for an active pharmaceutical ingredient (API), cetirizine dihydrochloride, in a drug formulation. ${ }^{19}$ Griffin and co-workers previously studied the dynamics of hyperpolarization $^{21}$ and the diffusion of polarization through peptide nanocrystals in a frozen solution. ${ }^{16}$

Note that, in comparison to conventional ${ }^{1} \mathrm{H}$ spin diffusionbased approaches, DNP requires a material stable under grinding, impregnation, and low temperatures. The advantages are, however, that there is a much larger polarization gradient induced by the DNP effect, which allows larger distances to be probed through spin diffusion. Also, higher sensitivity (in the microwaves on experiment) allows measurement of smaller targets with very low content (down to picomolar for some samples).

Here we present a general approach to model the relay of hyperpolarization in these experiments, and we provide a detailed analysis of four different archetypal geometries. Experimental validation is given with the determination of particle sizes in powdered histidine hydrochloride monohydrate, the pore lengths of mesoporous silica materials, and two domain sizes in two-component polymer film coatings. We show that with typical values of the relaxation times $T_{1}$ and diffusion rates $D$ in organic solids, the method is sensitive to architectures with sizes from $50 \mathrm{~nm}$ to $20 \mu \mathrm{m}$. We also provide analytical approximations that allow us to relate steady-state DNP enhancement values to the microarchitecture of the systems. In particular, we find that the analysis is also applicable to the nanoscale heterogeneity in the bulk polarizing solution itself.

\section{EXPERIMENTAL SECTION}

Sample Preparation. Powdered histidine hydrochloride monohydrate was obtained from Sigma-Aldrich and used without further purification. For DNP enhanced NMR experiments on powdered histidine hydrochloride monohydrate, the organic powder either was used as is or was finely ground by hand in an agate mortar and pestle for several minutes. Incipient wetness impregnation (IWI $)^{22}$ was used to uniformly wet the surface of around $30 \mathrm{mg}$ of the powdered solid with around $15 \mu \mathrm{L}$ of a solution containing $16 \mathrm{mM}$ state of the art biradical polarizing agent TEKPol in 1,1,2,2tetrachloroethane (TCE). ${ }^{23}$ Alternatively, a few milligrams of powder was dissolved in a solution containing $12 \mathrm{mM}$ biradical AMUPol in glycerol- $d_{8}: \mathrm{D}_{2} \mathrm{O}: \mathrm{H}_{2} \mathrm{O}(6: 3: 1)$ to obtain a homogeneous mixture. ${ }^{24}$ Samples were then transferred to a sapphire rotor, packed with a polytetrafluoroethylene (PTFE) insert, and capped with a PTFE or vespel cap.
The mesoporous materials were synthesized as described in the Supporting Information. For DNP enhanced NMR experiments on passivated mesoporous SBA-15 functionalized with a propylimidazolium group, between 30 and $40 \mathrm{mg}$ of the silica-based mesoporous material was wetted with between 10 and $20 \mu \mathrm{L}$ of a solution containing $16 \mathrm{mM}$ binitroxide polarizing agent TEKPol2 in 1,1,2,2-tetrachloroethane solution. ${ }^{25}$

Coated pellet samples, consisting of a microcrystalline cellulose (MCC) core, spray coated with a film of ethyl cellulose (EC) and hydroxypropyl cellulose (HPC) in a ratio of 70/30, were provided by AstraZeneca. For DNP enhanced NMR experiments, about $15 \mu \mathrm{L}$ of $16 \mathrm{mM}$ biradical solution TOTAPOL in glycerol- $d_{8}: \mathrm{D}_{2} \mathrm{O}: \mathrm{H}_{2} \mathrm{O}(6: 3: 1)$ was added to about $30 \mathrm{mg}$ of pellets. The wet sample was then mixed with a glass stirring rod and transferred to a sapphire rotor, packed with a PTFE insert, and capped with a PTFE cap.

DNP Enhanced NMR Spectroscopy. DNP enhanced solid-state NMR experiments were performed on wide bore $400 \mathrm{MHz}\left(B_{0}=9.4 \mathrm{~T}\right)$ Bruker Avance I and Avance IIIHD spectrometers both equipped with $263 \mathrm{GHz}$ gyrotrons, a lowtemperature cooling cabinet, and a triple-resonance $3.2 \mathrm{~mm}$ low-temperature magic angle spinning (MAS) probe. The sample temperature for DNP experiments was approximately $105 \mathrm{~K}$. The sweep coil of the main magnetic field $\left(\nu_{0}\left({ }^{1} \mathrm{H}\right)=\right.$ $400.432 \mathrm{MHz}$ ) was set so that microwave irradiation occurred at the same position as the $\varepsilon$ maximum for TOTAPOL. ${ }^{26}$ The estimated power of the microwave beam at the output of the waveguide was approximately $4-8 \mathrm{~W}$. The sample spinning rate $\left(\nu_{\text {rot }}\right)$ was $8000 \mathrm{~Hz}$. For all cross polarization (CP) experiments, the amplitude of the ${ }^{1} \mathrm{H}$ RF field was ramped during the contact time to improve efficiency (Scheme 1 ). ${ }^{2}$

Scheme 1. Saturation-Recovery ${ }^{1} \mathrm{H}-{ }^{13} \mathrm{C}$ CP Pulse Sequence Used for Acquisition of Spectra ${ }^{a}$

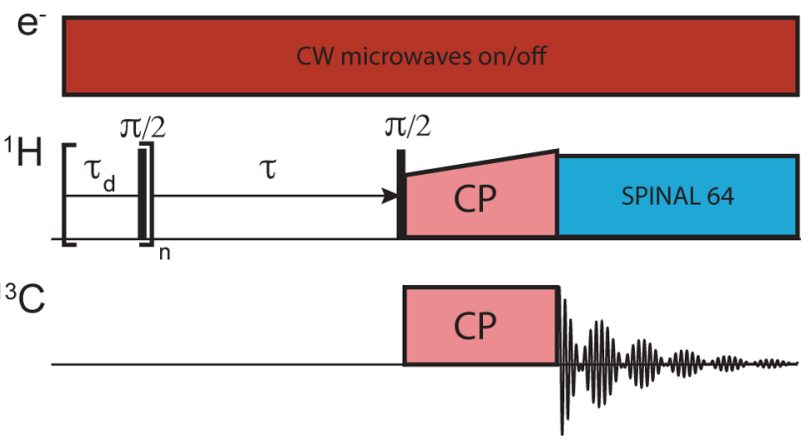

${ }^{a} \tau_{\mathrm{d}}$ was set to $20 \mathrm{~ms}$, and $n$ to $20 . \tau$ is the polarization delay.

Low-temperature solid-state NMR experiments without DNP were performed on a wide bore $500 \mathrm{MHz}$ (i.e., $B_{0}=$ $11.74 \mathrm{~T}$ ) Bruker III HD spectrometer equipped with a lowtemperature cooling cabinet and a triple resonance $3.2 \mathrm{~mm}$ lowtemperature magic angle spinning probe. The sample temperature was approximately $105 \mathrm{~K}$.

Numerical Simulations. Numerical simulations were performed using the Matlab differential equation solver "pdepe", which solves initial-boundary condition problems for partial differential equations in space $(x)$ and time $(t)$ domains. Two different differential equations were used for the cases where the microwave irradiation ( $\mu$ wave) is on or off. Initial and boundary conditions do not change between the $\mu$ wave on 
and off cases, whereas the local equilibrium polarization $P_{0}$ changes between $\mu$ waves on $\left(P_{0, \text { on }}\right)$ and $\mu$ waves off $\left(P_{0, \text { off }}\right)$ cases. The values of $T_{1}$ or $T_{\mathrm{B}}$ and $P_{0}$ where defined using the hyperbolic secant functions described in the main text and in the Supporting Information. For both $\mu$ waves on and off cases, numerical values of the polarization function that satisfy the differential equations were found, and the polarization was scaled by $|J(x)|$, where $|J(x)|$ is the Jacobian as described in Results and Discussion. The simulated signal was obtained by integration of the resulting polarization using a trapezoidal numerical integration. The simulated enhancement was calculated as the ratio of the integrated $\mu$ waves on and off signals. The normalization factors for the signals were obtained using the value of the signal at steady state. The Matlab code is available with the Supporting Information. Automatic fits were performed with Matlab using the least-squares method for both the enhancement and the buildup data.

\section{RESULTS AND DISCUSSION}

Spin Diffusion Relayed DNP. The basic principle behind the idea of measuring domain structures with DNP or PRE experiments is that the sample can be prepared in a way that one component contains a polarization agent, which will be referred to here as the source, while a second component is free of polarizing agents, which will be referred to as the target. When the source is hyperpolarized, polarization builds up quickly and then diffuses into the target through homonuclear proton spin-diffusion. The buildup of the polarization in the target thus depends on its size.

The observable buildup time of a compound resonance subject to spin diffusion in a saturation-recovery experiment can be quite different from the "intrinsic" buildup time. This effective buildup time has been previously referred to as $T_{\mathrm{B}}{ }^{28}$ $T_{1}{ }^{29} T_{1}{ }^{* 20}$ or $T_{\mathrm{DNP}}{ }^{30}$ Here it is essential to differentiate the intrinsic buildup time from both the $\mu$ waves on and $\mu$ waves off effective buildup times; the following notation will be used:

$T_{1}$ is the ${ }^{1} \mathrm{H}$ relaxation time measured during a saturationrecovery experiment on a pure dry solid.

$T_{B}$ is the effective ${ }^{1} \mathrm{H}$ buildup time measured when the sample is doped with paramagnetic species: $T_{\mathrm{B}, \mathrm{OFF}}$ in the absence of $\mu$ wave irradiations and $T_{\mathrm{B}, \mathrm{ON}}$ in the presence of $\mu$ wave irradiations.

In order to determine sizes quantitatively, we must develop a numerical model for the process, as described in the following.

The model used to describe the saturation-recovery experiments investigated here is based on a system containing at least two distinct domains. The source is selectively doped with a paramagnetic species, while the target remains undoped. The source also undergoes paramagnetic relaxation enhancement (PRE) induced by the presence of radicals. ${ }^{31} \mathrm{We}$ assume that the border between the two domains has a finite width, and we describe this interfacial region by a gradient function moving from pure source to pure target.

In the following, we model a system in which the recovery toward equilibrium for the two different components will not be a single monoexponential function with $T_{1 \text {,source }}$ and $T_{1, \text { target }}$. Rather, multiexponential buildup will be observed for both components if the domain sizes of the source and target are comparable to the distances over which spin diffusion can transport polarization on the $T_{1}$ time scales. We define the characteristic diffusion length as $\frac{x}{\sqrt{D T_{1}}}$ (see details in the Supporting Information). If the domain sizes are very small compared to the characteristic diffusion length, $L \ll \sqrt{\langle D\rangle\left\langle T_{1}\right\rangle}$, then both components will appear to relax toward equilibrium with the same buildup time, $T_{B}$. If the domain sizes are very large, $L \gg \sqrt{\langle D\rangle\left\langle T_{1}\right\rangle}$ compared to the diffusion length, then both components will appear to relax toward equilibrium with their intrinsic buildup times $T_{1 \text {,source }}$ and $T_{1, \text { target }}$. In the intermediate regime, where the domain sizes are comparable to the diffusion length, relaxation toward equilibrium will appear multiexponential and will be a function of the size and geometry of the domains. It is this effect that we exploit here. This basic principle of spin diffusion relayed relaxation has been introduced and used widely in the past in both solids and solutions. ${ }^{16-20,29,32-41}$ One of the particularities here is that we introduce differential relaxation into a normally diamagnetic sample by selective doping of one domain, and that we can then determine geometries by comparing polarization dynamics in experiments that are otherwise identical except for the initial conditions of the value of $T_{1}$ in the dopant (which corresponds to relayed-PRE ${ }^{20}$ ) or, here, by changing the equilibrium polarization of the dopant with DNP.

We also remark that recently there has been considerable interest in developing computational models for spin diffusion $^{42-47}$ and for different aspects of spin dynamics specifically in DNP experiments. ${ }^{46,48}$ Some very interesting work has been done to include the role of the depolarization induced by the biradicals under the MAS rotation. ${ }^{49,50}$ However, because we are concerned here with length scales beyond $\sim 50 \AA$, we adopt a method that is based solely on transport by spin diffusion. ${ }^{16,17}$

Numerical Models for Spin Diffusion Relayed DNP. The polarization dynamics is described by the following differential equation, where we assume the transfer of polarization behaves like thermal or other classical diffusion processes that follow Fick's second law: ${ }^{8,51,52}$

$$
\frac{\partial P(x, t)}{\partial t}=D(x) \cdot \Delta P-\frac{P(x, t)-P_{0}(x)}{T_{1}(x)}
$$

where $x$ is the position vector distance from the border between source and target in $\mu \mathrm{m}, t$ the time in seconds $(0<t<\infty), P$ the instantaneous polarization (arbitrary units), $P_{0}$ the local equilibrium polarization, $D$ the diffusion rate at position $x, T_{1}$ the longitudinal relaxation time at position $x$, and $\Delta P$ the Laplacian of the polarization whose formal expression depends on the symmetry of the system (see details in the Supporting Information).

In practice, depending on the nature of the physical interface between the source and the target, the thickness of the border will vary, as discussed later in the text. We consider two different functions for the change in NMR parameters at the interface. The first is the case for an interface that is a step function, and where the NMR properties then change as $1 / r^{6}$ beyond the interface (function described in detail in the Supporting Information). The second would be appropriate when the interface itself extends over a range of distance and where we consider that the gradient between the components at the crossover follows (for example) a hyperbolic tangent function, as shown in Figure 1. When the border is only $2 \mathrm{~nm}$ thick, the $1 / r^{6}$ dependence is most appropriate, but the shape of the function has little or no effect on the observables because the width of the objects considered here is on the order of micrometers. For objects for which the border is significantly thicker, the hyperbolic secant is more appropriate to describe a 
(a)

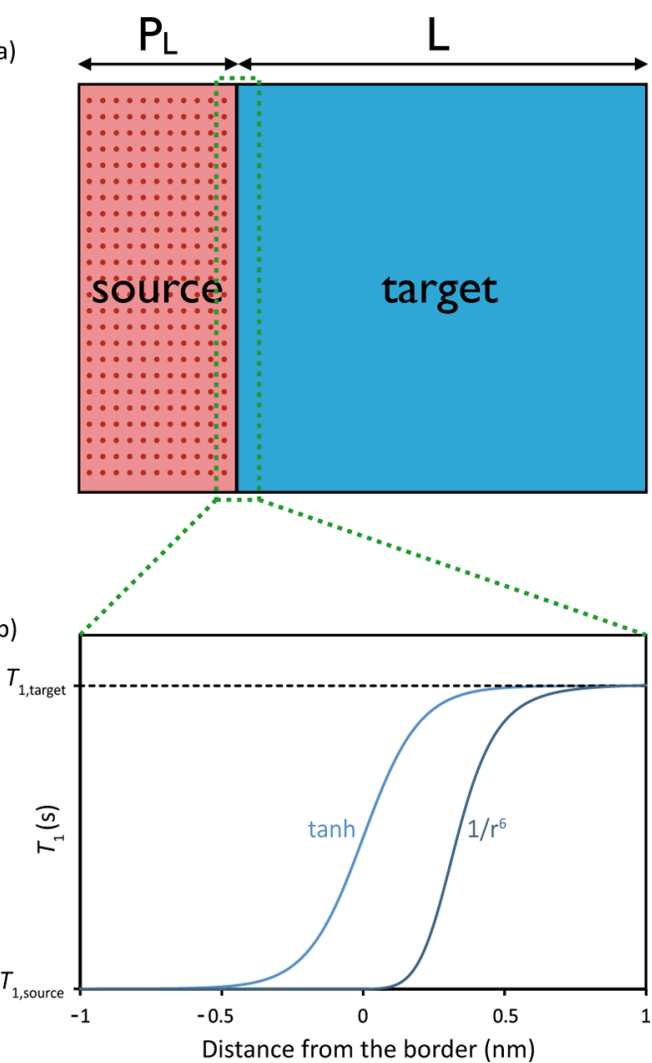

(c)

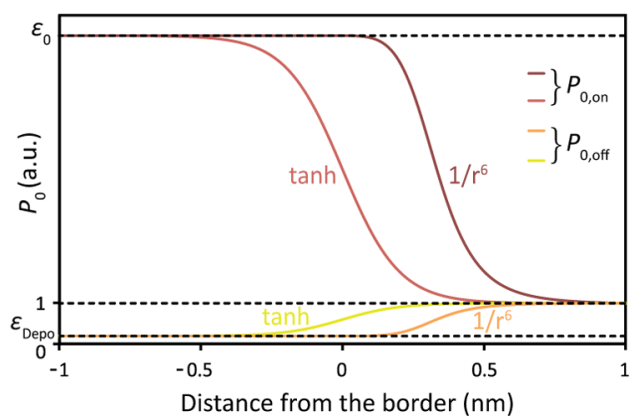

Figure 1. (a) Schematic representation of a system containing a source and a target. The source has a length $P_{\mathrm{L}}$ and is doped with, for example, organic radicals, whereas the target has a length $L$ and remains undoped. Red dots represent schematically the presence of radicals. Hyperbolic tangent (tanh) and $1 / r^{6}$ functions describe in panel $\mathrm{b}$ the relaxation time $T_{1}$ and in panel $\mathrm{c}$ the local equilibrium polarization $P_{0}$ as a function of the distance from the border.

gradient of radicals. For the sake of convenience, we chose to use here the hyperbolic secant function to describe the transition at the interface. However, as required, different functions could be used for the three variables, which might more accurately reproduce the physical and spatial dependencies of the interactions. The hyperbolic secant functions are described in detail in the Supporting Information.

Equation 1 does not have a general analytical solution. Under certain approximations, we can find some general analytical solutions as described in the Supporting Information. Here, we resort to numerical solutions with the following boundary conditions:

$$
P(x, 0)=0
$$

$$
\left(\frac{\partial P(x, t)}{\partial x}\right)_{x_{0}}=0
$$

where $x_{0}$ corresponds to the position at the extremity of the system. The first condition corresponds to no initial polarization inside the system (for saturation-recovery experiments), and the second corresponds to the absence of polarization flux at the edge of the system. The second condition implies no flux of polarization at the center of the target nor at the edge of the source. Notably we model the entire system as a single continuous object, and we do not need to partition the system (as long as the numerical solutions converge). Also, the boundary conditions reflect the fact that the system is periodic, i.e. each unit feels the presence of a neighbor.

Small Targets and Homogeneous Bulk Solutions. As shown in Figure 2a, a solution containing dissolved radicals can already be described using this general formalism. In this case the average distance between sources (where each radical molecule acts as a polarization source) is short enough that spin diffusion equalizes the polarization throughout the sample on a time scale short compared to the characteristic buildup times. This case corresponds to the situation of a bulk polarizing solution, e.g. containing a biradical such as TEKPol or AMUPol.

In this case, the average distance between (bi)radicals is defined as twice the Wigner-Seitz radius: ${ }^{53}$

$$
\bar{d}=2 \lambda=2\left(\frac{3}{4 \pi C N_{\mathrm{A}}}\right)^{1 / 3}
$$

where $C$ is the concentration of DNP active radicals and $N_{\mathrm{A}}$ is Avogadro's number. For a $16 \mathrm{mM}$ solution of biradical, if we assume all the radicals are DNP active for the sake of simplicity (though some fraction is probably not active, which would lead to a lower concentration of sources than relaxation sinks), we find a distance of $5.8 \mathrm{~nm}$. With a diffusion constant of $10^{-4}$ $\mu \mathrm{m}^{2} \mathrm{~s}^{-1}$, the volume between two radicals is polarized by spin diffusion in less than $10 \mathrm{~ms}$. This quasi-instantaneous spin diffusion leads to the observation of a monoexponential buildup for a bulk solution of radicals, despite the fact that the source and target areas have different intrinsic $T_{1}$. However, very importantly, we find here that a significant part of the solvent is mostly polarized by relayed transfer; therefore, the concentration of radicals does influence the observed DNP buildup time of the system. This has also been postulated very recently in simulations by Mentink-Vigier et al. ${ }^{48}$

As illustrated in Figure 2a, we define a volume close to the radical, $V_{\mathrm{Q}}$, in which ${ }^{1} \mathrm{H}$ spins undergo strong paramagnetic interaction because of the hyperfine coupling between nuclear and electron spins which leads to, for example, large anisotropic shifts or enhanced relaxation and quenching of the nuclear polarization. The large shift anisotropy means that spins inside $V_{\mathrm{Q}}$ communicate with each other much less efficiently, and that the nuclear spin diffusion is reduced (as an illustrative example, $D$ might decrease from values around $10^{-4} \mu \mathrm{m}^{2} \mathrm{~s}^{-1}$ at the border between $V_{\mathrm{Q}}$ and $V_{\mathrm{P}}$ to about $0 \mu \mathrm{m}^{2} \mathrm{~s}^{-1}$ at the location of the electron, see the Supporting Information). Additionally, we assume that spins inside $V_{\mathrm{Q}}$ do not produce any observable signal because of relaxation, but also because of the large anisotropic interaction that spread the resonances over a wide spectral window. We then define a volume, $V_{\mathrm{P}}$, in which ${ }^{1} \mathrm{H}$ spins are considered to be directly polarized by the radicals. Finally, as shown in Figure $2 \mathrm{a}$, the remaining volume, $V_{\mathrm{SD}}$, is 


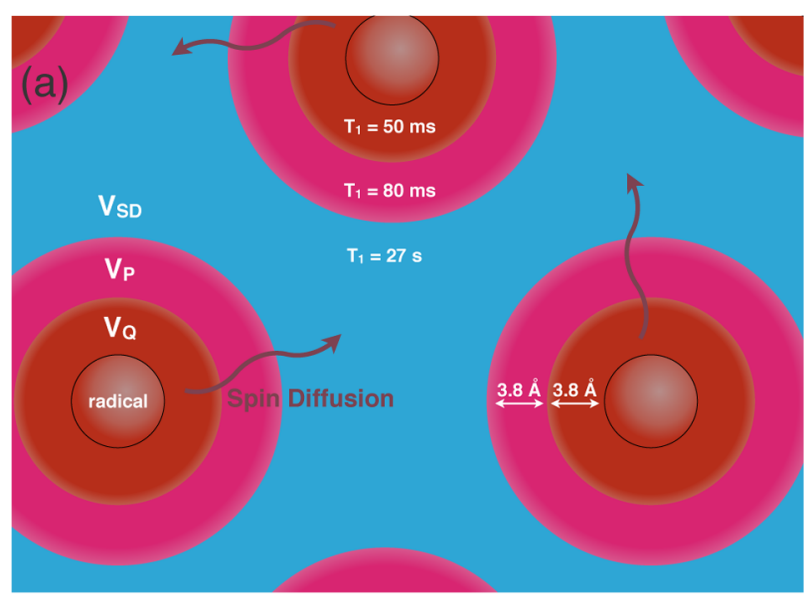

(b)

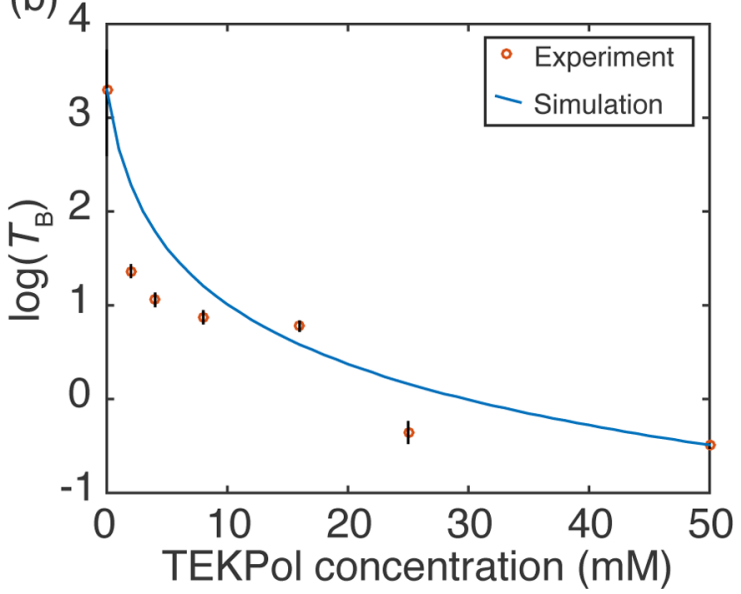

Figure 2. (a) Schematic representation of a bulk radical solution (small target) system. The different components are described in the main text. (b) Experimental ${ }^{1} \mathrm{H}$ buildup time $T_{\mathrm{B}}$ as a function of the concentration of TEKPol in a frozen solution of TCE at $105 \mathrm{~K}$, with 8 $\mathrm{kHz}$ sample spinning recorded on a $500 \mathrm{MHz}$ spectrometer, using saturation-recovery and determined using a monoexponential fit. All samples were degassed using 5 insert-eject cycles prior to measurement to minimize additional paramagnetic relaxation due to dissolved oxygen. $^{54}$ The solid blue line is the result of a numerical simulation for a source and target according to panel a for which at each concentration the buildup was simulated and then fit to a monoexponential. In the simulations, the system was assumed to be three-dimensional. $T_{1, \mathrm{VSD}}$ was measured to be $27 \mathrm{~s}$ (in pure TCE). The size of the volumes $V_{\mathrm{Q}}$ and $V_{\mathrm{P}}$ were both determined by fitting to be $3.8 \pm 0.1 \AA$. $T_{1, \mathrm{VQ}}$ and $T_{1, \mathrm{VP}}$ were determined by fitting to be $50 \pm 10$ and $80 \pm 10 \mathrm{~ms}$, respectively.

not directly polarized by the radicals. In this model the spins inside both $V_{\mathrm{P}}$ and $V_{\mathrm{SD}}$ are observable, and they are in contact by spin diffusion. Thus, in relation to Figure $1, V_{\mathrm{P}}+V_{\mathrm{Q}}$ constitute the source and $V_{\mathrm{SD}}$ is the target. The intrinsic $T_{1, \mathrm{VP}}$ and $T_{1, \mathrm{VQ}}$ of spins contained in $V_{\mathrm{P}}$ and $V_{\mathrm{Q}}$ are not directly measurable because we do not know a priori the ratio between $V_{\mathrm{P}}, V_{\mathrm{Q}}$, and $V_{\mathrm{SD}}$ in a given sample.

In Figure 2 , to determine $T_{1, \mathrm{VP}}$ and $T_{1, \mathrm{VQ}}$, we measure the buildup time $T_{\mathrm{B}}$ of a series of samples without $\mu$ wave irradiations and with different radical concentrations, for TEKPol in TCE at $105 \mathrm{~K}$. The data are then fit using the numerical model described above. The best fit between simulated and experimental buildup times was found for $V_{\mathrm{Q}}$ $=V_{\mathrm{P}}=3.8 \pm 0.1 \AA$, $T_{1, \mathrm{VQ}}=50 \pm 10 \mathrm{~ms}$, and $T_{1, \mathrm{VP}}=80 \pm 10$ $\mathrm{ms}$, as shown in Figure $2 \mathrm{~b}$. We note that an analogous model was tested, for which the diffusion rate was constant throughout the system $\left(D=10^{-4} \mu \mathrm{m}^{2} \mathrm{~s}^{-1}\right)$ which gave slightly worse fits with only slightly different $T_{1}$ values (see the Supporting Information).

The overall observed buildup time in the polarizing solution varies as a function of concentration because the volume $V_{\mathrm{SD}}$, which has the intrinsic buildup time of the pure solvent (here $T_{1, \mathrm{VSD}}$ for pure TCE is measured to be $27 \mathrm{~s}$ at $500 \mathrm{MHz}$ ), increases as the radical concentration decreases. Because spin diffusion over this length scale is much faster than $T_{1, \mathrm{VSD}}$, we therefore observe a single monoexponential buildup time for the sample which is simply a volume weighted average of the three intrinsic buildup times (here $T_{1, \mathrm{VSD}}=27 \mathrm{~s}, T_{1, \mathrm{VP}}=80 \mathrm{~ms}$ and $T_{1, \mathrm{VQ}}=50 \mathrm{~ms}$ ) and this explains the typically observable the signal buildup time constants of about 2-3 s typically observed in bulk solutions of $16 \mathrm{mM}$ biradicals at around 105 $\mathrm{K}^{23-25,55,56}$ The dependence of the experimental buildup time on residual volume was previously reported by Lange et al. ${ }^{57}$ in the context of the evaluation of quenching factors.

We note that we would expect this type of behavior for any kind of formulation in which the average closest distance between two radicals is smaller than the characteristic diffusion length (here $\sqrt{\langle D\rangle\left\langle T_{1}\right\rangle}=50 \mathrm{~nm}$ for TCE).

Large Targets and Heterogeneous Mixtures. We now move on to consider systems in which the dimension of the target $L \approx \sqrt{\langle D\rangle\left\langle T_{1}\right\rangle}$. In this case the polarization dynamics will be characteristic of the length and the geometry of the source and the target. In the following we show how to predict these dynamics numerically using Equations $1-3$. To do this we need to specify the spatial dependence of $T_{1}$, the local equilibrium polarizations, the diffusion constant, and the effect of depolarization.

Note that, because we will now study the diffusion of polarization on a scale much larger than in the "small targets" section above, we will now refer to the radical-solvent solution as a single source, without considering the $V_{\mathrm{P}}$ or $V_{\mathrm{SD}}$ volumes further. Because the source is now a mixture of radicals and solvent, its effective buildup time, which depends on the concentration of radicals, will be referred to as $T_{\mathrm{B} \text {,source }}$.

The spatial dependence of $T_{1}$ as it changes from the source to the target has been described above and is shown in Figure 1 for $r^{-6}$ and hyperbolic tangent functions. As before, the presence of radicals in the system will induce some signal quenching and/or depolarization in the source. ${ }^{22,50}$ Notably, for cross-effect biradicals, depolarization occurs in the source, and this will lead to a decrease in the source signal, typically around a factor 0.5 at $8 \mathrm{kHz}$ spinning rate at $9.4 \mathrm{~T}$ and 105 $\mathrm{K}^{49,50}$ We remark that depolarization does not play a role in the simulated buildup time for the small target regime described above, but it will influence the behavior in large targets.

The local equilibrium polarization $\left(P_{0}\right)$ is the polarization that each voxel would reach at infinite time if no spin-diffusion occurs. In the absence of microwaves, polarization will tend to recover in the target toward the Boltzmann polarization which we take to be $P_{0, \text { off }}=1$. In the source, however, depolarization may occur in the case of a biradical under MAS at rate $\nu$, and the polarization tends to a lower value of the local equilibrium polarization $P_{0, \text { off }}(\nu)=\varepsilon_{\text {Depo }}(\nu)$, where

$$
\varepsilon_{\text {Depo }}(\nu)=\frac{P_{0, \text { off }}(\nu>0)}{P_{0, \text { off }}(\nu=0)}
$$


where $P_{0, \text { off }}(\nu>0)$ and $P_{0, \text { off }}(\nu=0)$ are the $\mu$ waves off local equilibrium polarizations of the source for spinning and static samples, respectively. In the simulations, we will always take $\varepsilon_{\text {Depo }}=0.5$ in line with values found in the literature. ${ }^{50}$

In the presence of $\mu$ waves, the local equilibrium polarization within the source is greatly increased, such that $P_{0 \text {,on }}=\varepsilon_{0} P_{0 \text {,off }}$ where $\varepsilon_{0}$ is the DNP enhancement factor of the source in the absence of spin diffusion.

We now note that the steady-state source polarization does not necessarily reach the local equilibrium polarization in the presence of spin diffusion from the source to the target. For example, the source polarization close to the interface is higher than the local equilibrium polarization in the absence of $\mu$ waves because polarization will diffuse from the target to the interface to compensate MAS driven depolarization. On the other hand, diffusion from the source to the target in the presence of $\mu$ waves prevents the source from reaching its equilibrium value. With the typical sizes and diffusion coefficients that we consider here, where the sizes of the source are much larger than the diffusion length, for simplicity we will consider the polarization to be equal to the local equilibrium polarization throughout the source. Thus, the depolarization coefficient defined by Vega and co-workers ${ }^{49}$ because the ratio of signals is quite close that defined as the ratio of polarizations (more details in the Supporting Information).

Within the target, the equilibrium polarization will decay rapidly to $P_{0, \text { on }}=1$ as the direct DNP effect drops off according to Figure 1, and we choose to model this for simplicity with the same hyperbolic tangent function as used for the changes in $T_{1}$ (Figure 1c).

The value of the spin diffusion coefficient $D$ may vary from the source to the target as the proton concentration changes. Because the diffusion coefficient is thought to vary with $\lambda^{-4}$ where $\lambda$ is the Wigner-Seitz radius between protons, we then chose to scale the diffusion coefficient with $C^{4 / 3}$ where $C$ is the ${ }^{1} \mathrm{H}$ concentration. ${ }^{16,18}$ Moreover, depending on if the sample is amorphous or crystalline and on the temperature, the diffusion rate may vary. In the following, we will use common values of the diffusion rates reported in the literature for organic solids.

The thickness of the domains also affects spin diffusion dynamics. Indeed, the reservoir of polarization in both domains is not infinite, and for example if there is only a thin layer of source compared to the size of the target this would lead to a depletion of the source under $\mu$ wave irradiations and less hyperpolarization of the target. The source polarization then does not reach $\varepsilon_{0} P_{0, \text { off }}$. Analogously, in the absence of $\mu$ waves, a thin source compared to the size of the target would lead to an increase in the source polarization, and the polarization in the source will not reach the $P_{0, \text { off }}(\nu)=\varepsilon_{\text {Depo }}(\nu)$ level, as it would for an undoped sample. These limiting cases may be of interest in certain cases, and particularly for applications in dissolution $\mathrm{DNP},{ }^{58}$ but we will not consider them further here, where we look at cases where the source is always much larger than the diffusion length.

In the following we present examples of polarization dynamics characteristic of different target geometries.

Polarization Dynamics for Different Architectures. Figure 3 shows the three different archetypal geometries that will be considered and compared to experimental measurements.

The formalisms developed here can be adapted to any geometry, and they are applicable to both DNP and PRE experiments. The three examples we use are chosen because
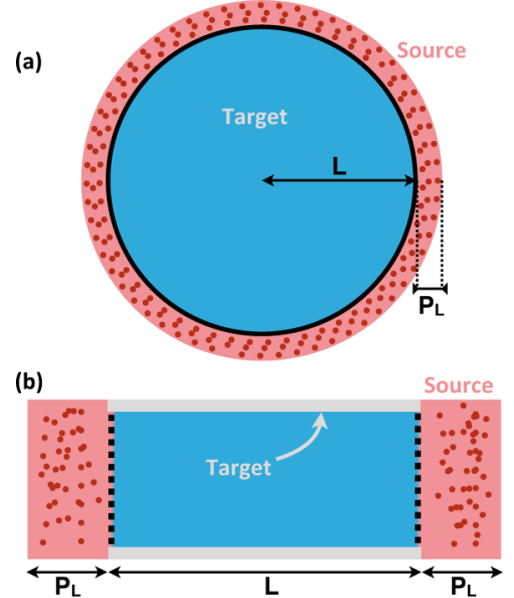

(c)

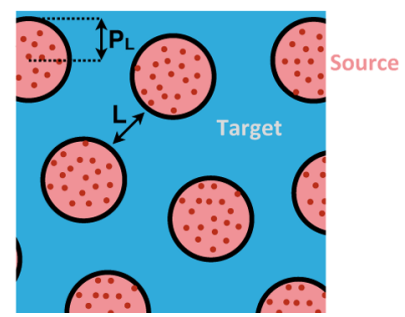

Figure 3. Schematic representation of (a) a spherical organic microcrystal coated with a radical containing solution; (b) a cylinder of pure solvent of length $L$, which is polarized by radical solution at the ends of the cylinder (this model corresponds for example to a solvent filling the pores in a mesoporous material, and where the radical does not enter the pores); and (c) an organic film containing hydrophilic domains (pink) that are doped with radicals and hydrophobic domains (blue) that remain undoped. Red dots indicate schematically the presence of radical molecules. In cases a and c, the radical is separated from the target because they are two different compounds: a solid line separates the two domains. In case b, the target can be either atoms on the wall of the pore or the proton atoms of the pure solvent inside the pore: a dashed line separates the pure solvent (blue) from the radical containing solvent (pink).

they correspond to common systems of experimental interest. The first system considered is a prototypical organic microcrystal or microparticle. The particle is assumed to be spherical (Figure 3a), and hyperpolarization diffuses into the particle from a layer of impregnating solvent. This corresponds to the situation for ordinary organic powders obtained by grinding and impregnation with a radical containing nonsolvent solution, ${ }^{17,19}$ or for a frozen suspension of particles. ${ }^{16}$

The second system is considered to be a prototypical pore, which we model as a cylinder (Figure $3 b$ ) into which the polarization diffuses from the outside. Because polarization has no known associated viscosity, we assume the diffusion of polarization to be constant over the sample. This model corresponds for example to a solvent filling the pores in a mesoporous material and where the radical does not enter the pores. $^{59,60}$ The solvent is pure inside the pore and doped with radical outside the pore.

The third system is prototypical mixture of polymers, where a soluble polymer domain is doped with radical, and insoluble domains remain radical free (Figure $3 \mathrm{c}$ ). ${ }^{20}$ We model this as a one-dimensional network.

Apart from the different symmetries of these geometries, the main difference between them will be the typical sizes and the relative thickness of the border between the source and the 
target. For example, in the cases of Figure 3a,c, the source and the target are two distinct compounds, and the border is welldefined. Whereas in Figure $3 \mathrm{~b}$, the transition from doped to undoped solvent is probably more gradual. ${ }^{59,60}$

The influence of the simulation parameters on the $\mu$ waves on polarization and on the enhancement are described in the Supporting Information. We use two types of border: one thin $(2 \mathrm{~nm})$ and one thick $(200 \mathrm{~nm})$. The border defines the area (or volume) in which the parameters described above transition. For both thick and thin border systems, the polarization is propagated numerically through the target until the steady state is reached. The resulting spatial evolution of the polarization as a function time for a spherically symmetric system is plotted in Figure 4.

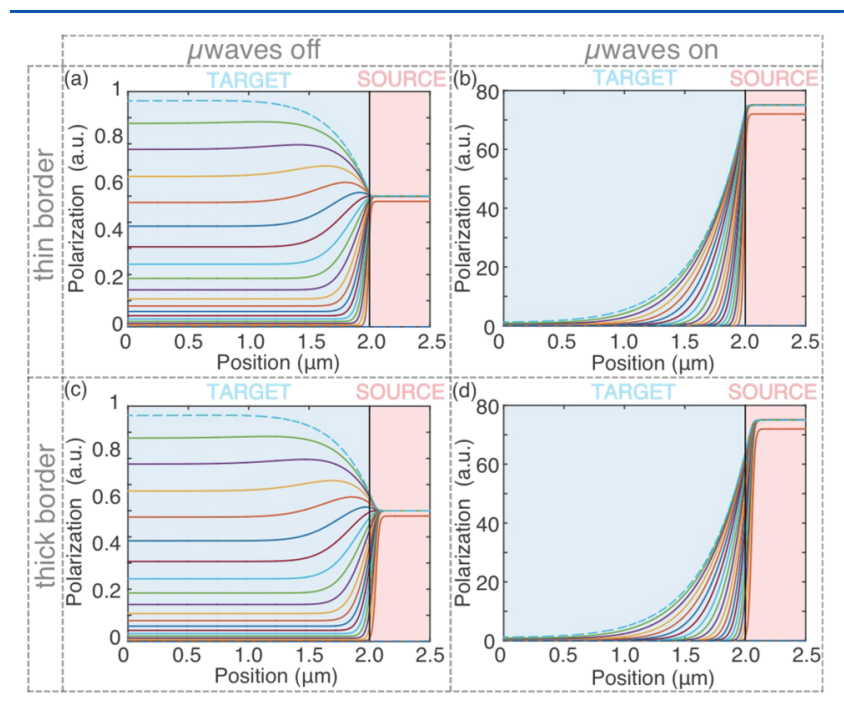

Figure 4. Simulations of polarization as a function of the position for a spherically symmetric system in (a, c) absence and (b, d) presence of microwave irradiation, for different times from $t=0$ to $200 \mathrm{~s}$ (where a steady state is reached, dashed light blue lines) with exponential time increment. Panels a and b have a thin border $(2 \mathrm{~nm})$ whereas $c$ and $d$ have a thick border $(200 \mathrm{~nm})$. The source is shown in red, whereas the target is shown in blue. In both cases, $L=2 \mu \mathrm{m}, T_{\mathrm{B}, \text { source }}=100 \mathrm{~ms}$, $T_{1, \text { target }}=100 \mathrm{~s}, \varepsilon_{\text {Depo }}=0.5, \varepsilon_{0}=150, D_{\text {target }}=D_{\text {source }}=1.0 \times 10^{-3}$ $\mu \mathrm{m}^{2} \mathrm{~s}^{-1}$, and $P_{\mathrm{L}}=0.5 \mu \mathrm{m}$.

The polarization is then integrated over the desired region, giving a signal:

$$
S_{\text {on/off }}(t)=\frac{1}{V} \int_{V} P_{\text {on } / \text { off }}(x, t) \cdot|J(x)| \mathrm{d} x
$$

where $S_{\text {on }}$ and $S_{\text {off }}$ correspond to the signals with and without microwave irradiation, respectively; $P_{\text {on }}$ and $P_{\text {off }}$ correspond to the polarization with and without microwaves irradiation; and I $J(x) \mid$ corresponds to the Jacobian determinant: $|J(x)|=1$ for linear symmetry, and for spherical symmetry, after integration on the angular coordinates, we have $|J(x)|=4 \pi x^{2}$. (The adaptation to other geometries can be done, for example, for a system where spin diffusion can occur in two dimensions: the Jacobian thus has to be equal to $2 \pi x$. Other changes also have to be made in the diffusion eq 1 , as described at the end of Results and Discussion.)

Archetypal signal buildup curves for the target for $\mu$ wave on and off are plotted in Figure 5a for the thin and thick border cases. We immediately note that the recovery is accelerated in the presence of $\mu$ waves, and this is the primary signature of relayed DNP.

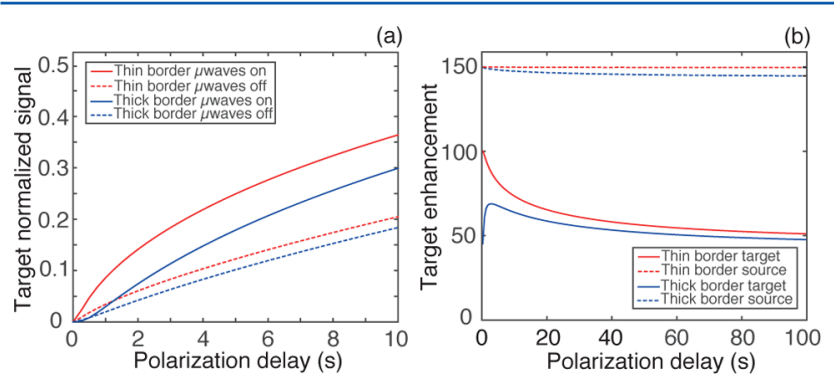

Figure 5. (a) Simulations of normalized target signal buildup as a function of time in absence and in the presence of microwave irradiation for a thin border (red) and a thick border (blue). (b) Simulated enhancement as a function of time in the target (solid lines) and in the source (dashed lines) for a thin border (red) and a thick border (blue). In all simulations, $L=2 \mu \mathrm{m}, T_{\mathrm{B}, \text { source }}=100 \mathrm{~ms}, T_{1, \text { target }}=$ $100 \mathrm{~s}, \varepsilon_{0}=150, D_{\text {target }}=D_{\text {source }}=1.0 \times 10^{-3} \mu \mathrm{m}^{2} \mathrm{~s}^{-1}$.

We then note that the main difference between the thin and thick border cases occurs at early times. This is not surprising, because at early times the target hyperpolarization is located at the border between the source and the target. Its thickness then determines the intensity of the signal at early times.

The enhancement as a function of time can be then trivially calculated:

$$
\varepsilon(t)=\frac{S_{\mathrm{on}}(t)}{S_{\mathrm{off}}(t)}
$$

Figure $5 \mathrm{~b}$ shows enhancement versus time for the same cases. We remark that the enhancement varies significantly with time, and this is a key signature of heterogeneous polarization and relayed transfer. Indeed, if the sample polarization is homogeneous, then spin diffusion will not cause any change in local polarization, and enhancement would be constant as a function of the polarization time.

For a system with a source and a large target, the normalized signal follows a multi (or stretched) exponential buildup:

$$
S(t)=1-\mathrm{e}^{-\left(t / T_{\mathrm{B}}\right)^{\beta}}
$$

where $T_{\mathrm{B}}$ is the effective buildup time with or without $\mu$ wave irradiation. In the case of a homogeneous system, spin diffusion has no effect on local polarization, and the signal buildup will be monoexponential $(\beta=1)$. The buildup time of the signal buildup in $\mu$ wave on $\left(T_{\mathrm{B}, \mathrm{ON}}\right)$ and off $\left(T_{\mathrm{B}, \mathrm{OFF}}\right)$ is then the same, and so the enhancement is constant.

As mentioned earlier, the difference between thin and thick border cases has an effect on enhancement at early times, and Figure $5 \mathrm{~b}$ shows that this is predicted to lead to a completely different enhancement behavior. For the thin border case, the enhancement decreases monotonically as a function of time. The thick border case is significantly different with an increase in the enhancement at early times, followed by a decay.

The late stage decay of enhancement as a function of time can be explained physically as follows. With time, the target polarization builds up to 1 in the $\mu$ wave off case (without considering depolarization), whereas it is far from reaching $\varepsilon_{0}$ in the $\mu$ wave on case, because of longitudinal relaxation (see Figure $4 b, d)$. Thus, with time, the target polarization increases relatively more in the $\mu$ wave off than in the $\mu$ wave on case. This 
explains the late stage decay of the enhancement seen in both thick and thin border cases. The early time increase in enhancement seen for the thick border case is rationalized by looking at the source behavior in the border region, as described in detail in the Supporting Information.

In summary, in Figure $5 b$ we see that the nature of the border influences the behavior of the enhancement at early times and that the decay to a steady-state enhancement at later times in both cases is a universal signature of relayed spin diffusion.

We note in Figure 5a that the observable buildup time is always shorter for $\mu$ waves on than off. This is easily explained by the fact that the gradient of polarization between the source and the target is much larger with than without DNP. We also note that $T_{\mathrm{B}, \text { target }}<T_{1, \text { target }}$. This arises from the fact that spin diffusion, both in $\mu$ wave on and off cases, connects polarization between the source and the target and because $T_{\mathrm{B} \text {,source }}$ is here always shorter than $T_{1, \text { target; }}$ this accelerates the effective buildup time in the target. Specifically, fitting the buildup curves of Figure $5 \mathrm{a}$ to a stretched exponential, we find that $T_{\mathrm{B}, \text { target }}$ is always shorter than $42 \mathrm{~s}$, whereas $T_{1, \text { target }}=100 \mathrm{~s}$. An important consequence of this observation is that, because proportionally more spins are closer to the source in smaller objects, we predict shorter buildup times for smaller objects. Figure 6 shows simulations of the normalized signal buildup curves and enhancements of the target for a spherically symmetric object for different target sizes.
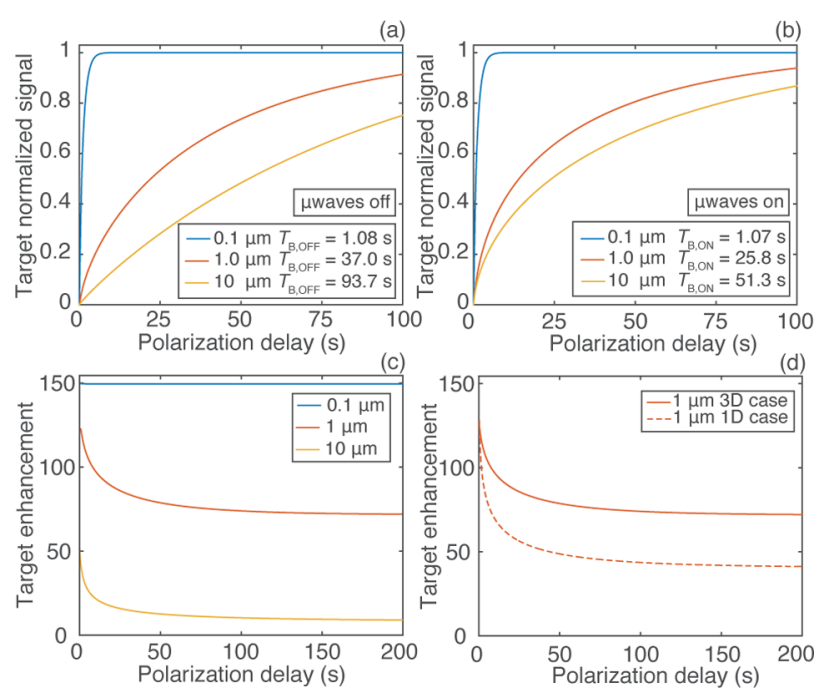

Figure 6. Spherical targets with a thin border. Simulations of normalized signal buildup curves for different target radii as a function of time for (a) $\mu$ waves off and (b) $\mu$ waves on. Buildup times $T_{\mathrm{B}}$ were determined from a fit to a stretched exponential. (c) Enhancement of the target as a function of time for different radii. (d) Enhancement of the target for a spherical and a linear geometry. In all simulations, $T_{\mathrm{B}, \text { source }}=100 \mathrm{~ms}, T_{1, \text { target }}=100 \mathrm{~s}, \varepsilon_{0}=150$, and $D_{\text {target }}=D_{\text {source }}=1.0 \times$ $10^{-3} \mu \mathrm{m}^{2} \mathrm{~s}^{-1}$.

In Figure $6 \mathrm{a}, \mathrm{b}$, as expected, the buildup time of the target is predicted to increase with the size of the object. The enhancement decreases with the size of the target, because relaxation in the target destroys the propagating hyperpolarization before it has time to spin diffuse through the whole target (Figure 6c). As shown in Figure 6d, we observe that the enhancement of a spherical object is higher, with all other parameters being identical, than the enhancement for a linear object being polarized from the ends. Indeed, with the same polarizing length $L$, the spherical symmetry offers less target volume to be polarized for unit of polarizing surface with respect to the linear symmetry. For a diffusion in two dimensions, we expect a curve in between the two shown.

Buildup Time and Steady-State Enhancement Behavior. As shown in Figure $6 \mathrm{~b}$, the buildup time depends on the size of the target, ranging from $T_{\mathrm{B} \text {,target }}=T_{\mathrm{B} \text {,source }}=3 \mathrm{~s}$ for an infinitely small target (which corresponds to a homogeneous system) and tends to $T_{1, \text { target }}$ for an infinitely large target. (The values of the $\beta$ coefficient of the stretched exponentials are shown in Figure S15.)

At long polarization times, a steady state is achieved in which relaxation exactly balances diffusion polarization flow into the target. The change in the steady-state enhancement as a function of the size of the object is shown in Figure 7. The
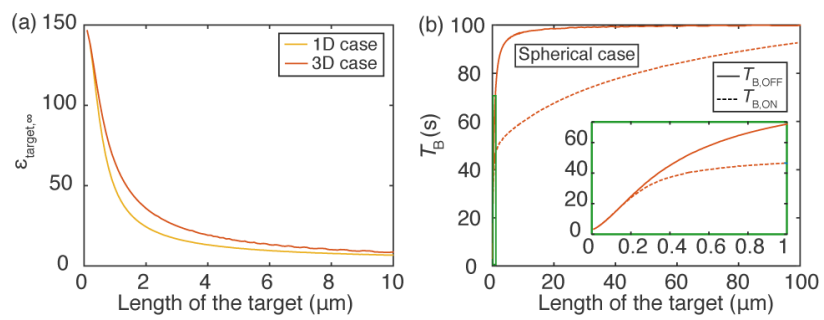

Figure 7. (a) Simulated enhancement of the target at steady state as a function of the size of the target for spherically and linearly symmetric objects with thin borders. (b) Simulated buildup times of the target as a function of the size of the target for a spherically symmetric object. In all simulations, $T_{\mathrm{B}, \text { source }}=3 \mathrm{~s}, T_{1, \text { target }}=100 \mathrm{~s}, \varepsilon_{0}=150$, and $D_{\text {target }}=$ $D_{\text {source }}=1.0 \times 10^{-3} \mu \mathrm{m}^{2} \mathrm{~s}^{-1}$.

great interest of the steady-state enhancement is that it does not depend on the nature of the border. As shown below, the steady-state enhancement is only a function of the geometry (linear, cylindrical, or spherical), the length $L$, the relaxation time $T_{1, \text { target }}$ and the diffusion rate $D_{\text {target }}$.

We fit the curves in Figure $7 \mathrm{a}$ with an expression derived from the steady-state solution to eq 1 :

$$
\varepsilon_{\infty}=1+(\mathrm{a}-1) \frac{2 \sqrt{b}}{L} \tanh \left(\frac{L}{2 \sqrt{b}}\right)
$$

for the $1 \mathrm{D}$ case and

$$
\varepsilon_{\infty}=1+(\mathrm{a}-1) \frac{3 \sqrt{b}}{L}\left[\operatorname{coth}\left(\frac{L}{\sqrt{b}}\right)-\frac{\sqrt{b}}{L}\right]
$$

for the $3 \mathrm{D}$ case where $\varepsilon_{\infty}$ is the steady-state enhancement of the target, $a$ and $b$ are fitting parameters, and $D$ and $T_{1}$ are the diffusion rate and relaxation time in the target, respectively. The reasoning behind the choice of these functions is given in the Supporting Information. By inspection, the fitted values of $a$ and $b$ are found to be

$$
a=\varepsilon_{0}
$$

where $\varepsilon_{0}$ corresponds to the enhancement of the source (which is constant) and

$$
b=D \cdot T_{1}
$$

A phenomenological relation between the steady-state enhancement and the size of the object can thus be determined as 


$$
\varepsilon_{\infty}=1+\left(\varepsilon_{0}-1\right) \frac{2 \sqrt{D \cdot T_{1}}}{L} \tanh \left(\frac{L}{2 \sqrt{D \cdot T_{1}}}\right)
$$

for the $1 D$ case and

$$
\varepsilon_{\infty}=1+\left(\varepsilon_{0}-1\right) \frac{3 \sqrt{D \cdot T_{1}}}{L}\left[\operatorname{coth}\left(\frac{L}{\sqrt{D \cdot T_{1}}}\right)-\frac{\sqrt{D \cdot T_{1}}}{L}\right]
$$

for the 3D case. Similar fitting was done for the cylindrical (or 2D) case, leading to a steady-state enhancement of

$$
\varepsilon_{\infty}=1+\left(\varepsilon_{0}-1\right) \frac{2 \sqrt{D \cdot T_{1}}}{L}\left[\frac{I_{1}\left(\frac{L}{\sqrt{D T_{1}}}\right)}{I_{0}\left(\frac{L}{\sqrt{D T_{1}}}\right)}\right]
$$

where $I_{0}$ and $I_{1}$ are the zero and first-order modified Bessel functions, respectively.

We can reinforce this observation by noting how the diffusion equation (eq 1 ) behaves at steady state. In the absence of depolarization, without microwave irradiation, $P_{0, \text { off }}=1$ in the target. With microwave irradiation, we also have $P_{0, \text { on }}=1$ in the target. Then, assuming that the diffusion and relaxation times are constant in the target (which is reasonable, e.g., for a border thickness of $2 \mathrm{~nm}$ ), for one-dimensional spin diffusion, the steady-state solution in the target is

$$
P(x, \infty)=A \mathrm{e}^{x / \sqrt{D T_{1}}}+B \mathrm{e}^{-x / \sqrt{D T_{1}}}+1
$$

where $A$ and $B$ depend on the boundary conditions (see details in the Supporting Information). This is in agreement with previous results. ${ }^{16,61} \mathrm{We}$ observe that the position dependence is scaled by the term $\sqrt{D T_{1}}$, and the polarization is in fact a function of the characteristic diffusion length $\frac{x}{\sqrt{D T_{1}}}$ as detailed in the Supporting Information. This observation rationalizes the phenomenological form of $b$ observed for the steady-state enhancement in eq $12 \mathrm{a}-\mathrm{c}$.

\section{EXPERIMENTAL RESULTS}

To support the predictions made above we have performed experiments on three prototypical systems.

Homogeneous Sample. The homogeneous case was verified with a sample of histidine dissolved in glycerol$d_{8}: \mathrm{D}_{2} \mathrm{O}: \mathrm{H}_{2} \mathrm{O}(60: 30: 10)$ with AMUPol as the biradical polarization source ${ }^{24}$ and recording the enhancement of the signals from histidine and glycerol. The prediction is that a homogeneous system displays a constant enhancement as a function of polarization time. As shown in Figure 8a, the histidine ${ }^{13} \mathrm{C}$ NMR peaks are much broader than the peaks observed when the crystalline histidine is impregnated with a radical solution remotely polarized (see Figure 10a). The signal buildup of histidine peaks was recorded with a CP saturationrecovery experiment. The $\mu$ waves on buildup time was the same as the solvent buildup time as shown in Figure $8 b$, confirming that the histidine, glycerol, water, and radical molecules are homogeneously mixed. As shown in Figure 8c, the enhancement of both histidine and glycerol was found to be constant over $15 \mathrm{~s}$, which confirms the prediction. The measurement error is relatively large for the histidine peaks because the signal-to-noise ratio was poor in the $\mu$ waves off spectra even with 2048 scans $(8.5 \mathrm{~h})$, due to low concentration of histidine.

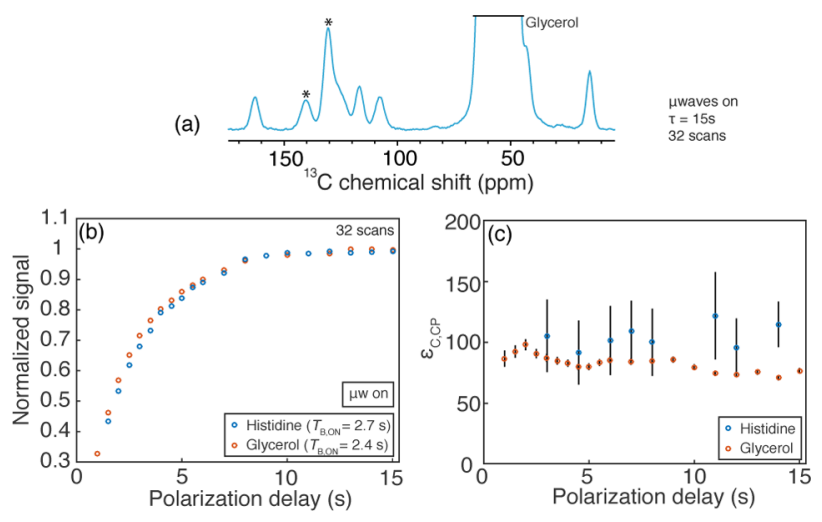

Figure 8. (a) ${ }^{13} \mathrm{C}$ CPMAS DNP enhanced solid-state NMR spectrum of histidine with microwave irradiation obtained at $105 \mathrm{~K}, 8 \mathrm{kHz}$ MAS, and $B_{0}=9.4 \mathrm{~T}$. For spectrum a, around $0.5 \mathrm{mg}$ of powdered histidine was dissolved in $100 \mu \mathrm{L}$ of glycerol- $d_{8}: \mathrm{D}_{2} \mathrm{O}: \mathrm{H}_{2} \mathrm{O} \quad(60: 30: 10)$ containing $12 \mathrm{mM}$ AMUPol. The polarization delay was set to $15 \mathrm{~s}$. (b) Normalized signal buildup with microwave irradiation of the histidine and glycerol peak areas as a function of the polarization delay and (c) the corresponding enhancement of the histidine and glycerol signals. The asterisks indicate spinning sidebands.

Heterogeneous Samples: Microcrystalline Powder. To confirm predictions of the simulations that signal buildup times depend on the size of the target in heterogeneous samples, ${ }^{13} \mathrm{C}$ detected ${ }^{1} \mathrm{H}$ buildup times of histidine signals were measured when the histidine was either dissolved in a radical containing solution, ground then impregnated with a solution, unground and impregnated, and for the dry powder alone. As shown in Figure 9, the buildup time clearly increases with the size of the particle, ranging for the $\mu$ waves on case from $2.7 \mathrm{~s}$ for the homogeneous solution to $435 \mathrm{~s}$ when the size of the particles is large, up to $1038 \mathrm{~s}$ for the dry powder which corresponds to the intrinsic $T_{1}$ of crystalline histidine. As predicted for heterogeneous systems, shorter buildup times are measured for $\mu$ waves on than for $\mu$ wave off for the impregnated powders.

Figure 10a shows the DNP enhanced spectrum from a sample of microcrystalline histidine ground for $5 \mathrm{~min}$ to reduce particle sizes and impregnated with 1,1,2,2-tetrachloroethane (TCE) containing $16 \mathrm{mM}$ TEKPol biradical. ${ }^{23}$ We notice that the histidine ${ }^{13} \mathrm{C}$ NMR peaks in this heterogeneous sample are much narrower than those in the homogeneous solution of Figure 8 , because the organic domains now remain crystalline. In Figure $10 \mathrm{~b}$ we show the normalized signal buildup of TCE as a function of the polarization delay with and without microwave irradiation, together with the prediction for the homogeneous solvent. Simulations are overlaid on the experimental data points. Analogously to the homogeneous case, the buildup time does not depend on the $\mu$ wave irradiations because the TCE domains contain homogeneously dispersed radicals. The enhancement of the TCE signal is thus found to be constant, as shown in Figure 10c. The buildup of the histidine peaks with and without microwave irradiation is shown in Figure 10d, together with the prediction for spherical domains of radius of $2.3 \mu \mathrm{m}$ (see figure caption for the other parameters), which is in good agreement with the size measured in scanning electron microscopy images (see the Supporting Information). The same system was also studied by DNP with unground particles, as shown in the Supporting Information, and a $9 \mu \mathrm{m}$ average particle radius was determined. 


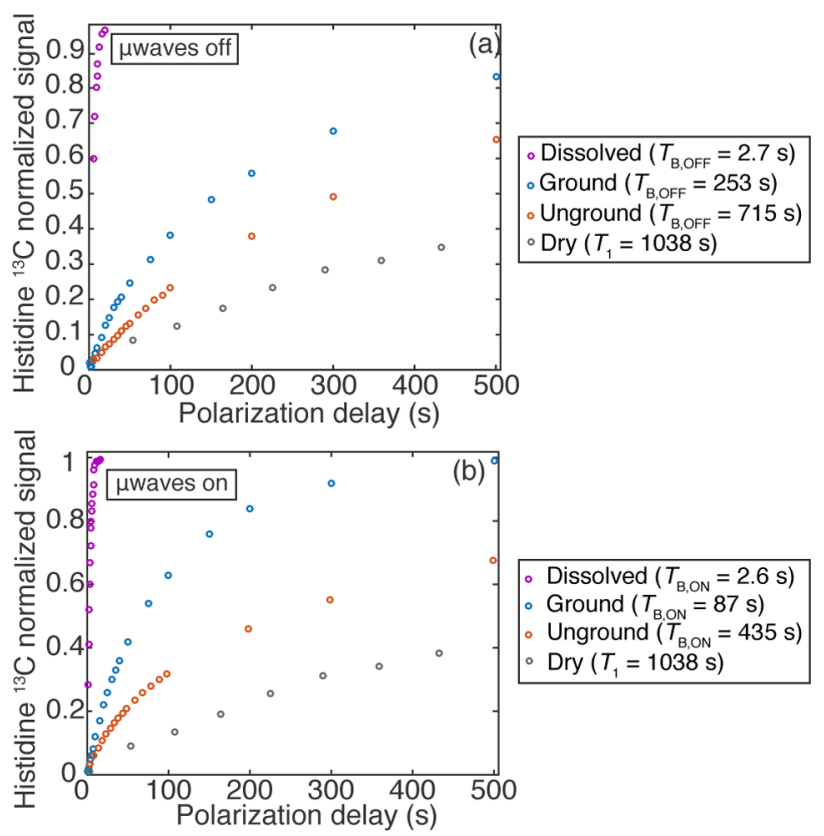

Figure 9. Signal ${ }^{1} \mathrm{H}$ buildup curves detected through the signal of the ${ }^{13} \mathrm{C}$ peaks in CPMAS NMR spectra recorded at $105 \mathrm{~K}, 8 \mathrm{kHz}$ MAS, and $B_{0}=9.4 \mathrm{~T}$ (a) without and (b) with microwave irradiation of either dry, ground, unground, or dissolved powders of histidine hydrochloride monohydrate. The dissolved sample was in glycerol$d_{8}: \mathrm{D}_{2} \mathrm{O}: \mathrm{H}_{2} \mathrm{O}(60: 30: 10)$ with $12 \mathrm{mM}$ AMUPol. The impregnation was done with 1,1,2,2-tetrachloroethane (TCE) containing $16 \mathrm{mM}$ TEKPol. All signals were normalized using the steady-state value from the exponential fits.

Heterogeneous Samples: Porous Materials. A mesoporous material MatImPh-dTMS was impregnated with a TCE solution containing TEKPol2, ${ }^{25}$ as shown in Figure 11.

Here we assume that the bulky radical ${ }^{25}$ cannot thoroughly enter the pore ${ }^{18,59,60}$ so that the silicon sites on the pore surface are polarized by spin diffusion through the solvent as illustrated in Figure 3. The enhancement behavior here is indeed found to be in extremely good agreement with the predictions of this model, including the details of the rising and then decaying enhancement for early polarization times, for a pore length of $0.75 \mu \mathrm{m}$.

Heterogeneous Samples: Polymer Composites. EC/ HPC coated microcrystalline cellulose (MCC) pellets were impregnated with $16 \mathrm{mM}$ TOTAPOL biradical in glycerol$d_{8}: \mathrm{D}_{2} \mathrm{O}: \mathrm{H}_{2} \mathrm{O}(60: 30: 10)$ solution, as shown in Figure 12.

Here we assume that the aqueous polarization source impregnates the soluble HPC domains, but that it does not penetrate the insoluble EC domains, which are thus considered as the target. The enhancement behavior here is again found to be in extremely good agreement with the predictions of this model, including the details decaying enhancement for early polarization times, for EC domains of $0.2 \mu \mathrm{m}$. This is in agreement with measurements made using NMR PRE type approaches. $^{20}$

Heterogeneous Samples: Colloidal Suspensions. Finally, insoluble EC Aquacoat nanoparticles were suspended in a solution of $16 \mathrm{mM} \mathrm{bCTbK}{ }^{23}$ in TCE/MeOH (80:20). The relayed DNP measurements shown in Figure 13 are in excellent agreement with a one-dimensional diffusion model with a diameter of $170 \mathrm{~nm}$, which is in good agreement with the 169 $\mathrm{nm}$ average measured by dynamic light scattering.
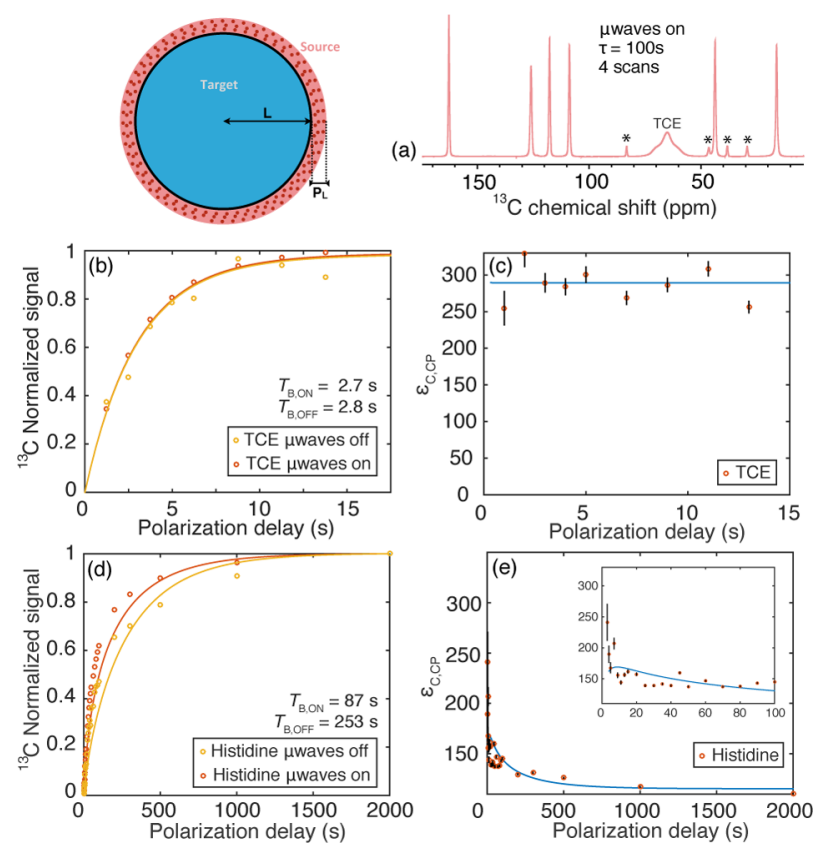

Figure 10. (a) ${ }^{13} \mathrm{C}$ CPMAS DNP enhanced solid-state NMR spectra obtained at $105 \mathrm{~K}, B_{0}=9.4 \mathrm{~T}$, and $8 \mathrm{kHz}$ MAS of microcrystalline histidine which was ground by hand for $5 \mathrm{~min}$ and impregnated with a solution of TCE containing TEKPol. (b) ${ }^{13} \mathrm{C}$ CPMAS normalized signal buildup of the TCE peaks with and without microwave irradiation. Normalization was performed using the value of the signals at steady state from a stretched exponential fit. (c) ${ }^{13} \mathrm{C}$ CP DNP enhancement of TCE peaks as a function of the polarization delay. (d) Signal buildup of histidine peaks with and without microwave irradiation as a function of the polarization delay. (d) ${ }^{13} \mathrm{C}$ CP DNP enhancement of histidine peaks as a function of the polarization delay. The histidine buildup points correspond to the average of the integrated areas of all the histidine ${ }^{13} \mathrm{C}$ peaks. Error bars were calculated by propagation of error using the noise levels of the spectra acquired with and without microwave irradiation as the standard deviation. In panels $\mathrm{d}$ and e, numerical simulations are overlapped for particle with spherical symmetry with $L=2.3 \mu \mathrm{m}$ (fitted), $T_{\mathrm{B}, \text { source }}=$ $2.7 \mathrm{~s}, T_{1, \text { histidine }}=1038 \mathrm{~s}, D_{\text {target }}=1 \times 10^{-3} \mu \mathrm{m}^{2} \mathrm{~s}^{-1}, D_{\text {source }}=1.3 \times 10^{-4}$ $\mu \mathrm{m}^{2} \mathrm{~s}^{-1}, P_{\mathrm{L}}=1 \mu \mathrm{m}$, and $\varepsilon_{\text {Depo }}=0.5$. The asterisks indicate spinning sidebands.

Size Distributions and the Correlation between Size and the Diffusion Constant. In the descriptions above we have assumed a single value of the particle sizes present in the samples. Of course, the same approach can be used to model the spin diffusion behavior in samples with particle size distributions by modeling the sums of the curves calculated above. For example, for the histidine crystals, a Weibull ${ }^{62}$ distribution might be considered as was done by Rossini et al. ${ }^{17,19}$ This might produce better agreement in the early time behavior, though there is a clear danger of overfitting. For the case of the EC suspensions in Figure 13, no significant improvement is seen if a Weibull distribution is used to fit the data, as shown in the Supporting Information. The only change is that the center of the distribution is slightly shifted $(190 \mathrm{~nm}$ instead of $170 \mathrm{~nm}$ ) because of the asymmetry of the Weibull distribution function.

Finally, we note that here $D$ is known to be typically between $0.2 \times 10^{-3}$ and $1.0 \times 10^{-3} \mu \mathrm{m}^{2} \mathrm{~s}^{-1}$ in most organic solids. ${ }^{63,64}$ In the examples above we have assumed values of $D$ most appropriate to each case on this basis, which allows us to deduce lengths from the buildup behaviors. As mentioned 

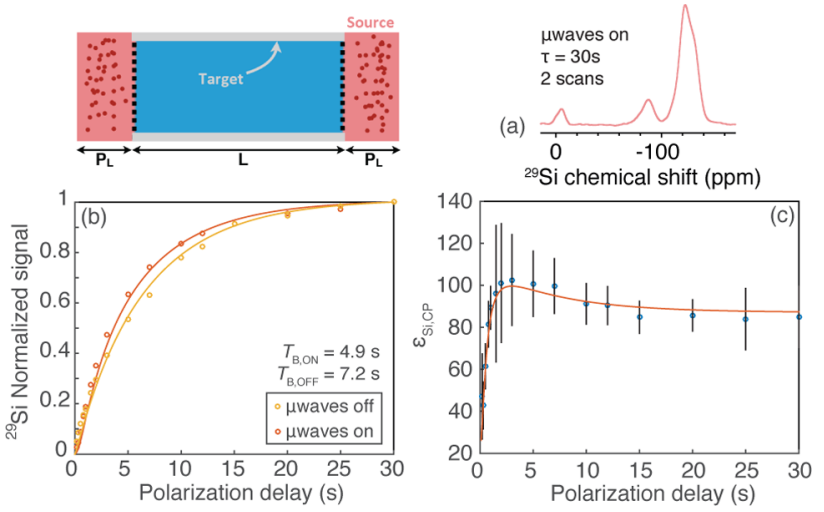

Figure 11. (a) ${ }^{29} \mathrm{Si}$ CPMAS DNP enhanced solid-state NMR spectra obtained at $105 \mathrm{~K}, B_{0}=9.4 \mathrm{~T}$, and $8 \mathrm{kHz}$ MAS of the mesoporous material MatImPh-dTMS impregnated with TCE containing $16 \mathrm{mM}$ biradical TEKPol2. (b) ${ }^{29} \mathrm{Si}$ CPMAS normalized signal buildup of the silicon peaks with and without irradiations. The signals were normalized to that of the longest polarization delay. (c) ${ }^{29} \mathrm{Si} \mathrm{CP}$ DNP enhancement of the ${ }^{29} \mathrm{Si}$ signal as a function of the polarization delay. In panels $b$ and $c$, numerical simulations are overlaid for onedimensional diffusion with the following parameters: $L=0.75 \mu \mathrm{m}$ (fitted), $T_{\mathrm{B}, \text { source }}=442 \mathrm{~ms}, T_{\mathrm{B}, \mathrm{TCE}}=8 \mathrm{~s}$, and $D_{\text {target }}=D_{\text {source }}=1.2 \times$ $10^{-4} \mu \mathrm{m}^{2} \mathrm{~s}^{-1}$.
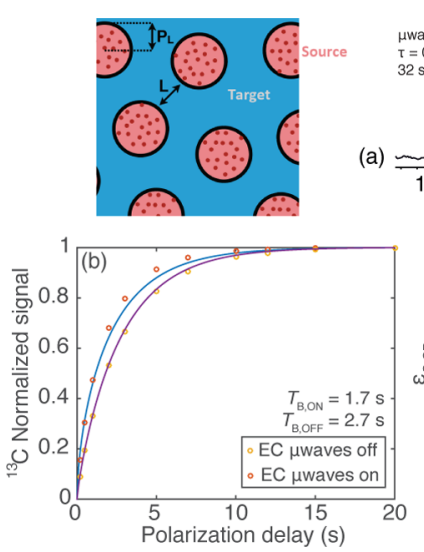

(a)
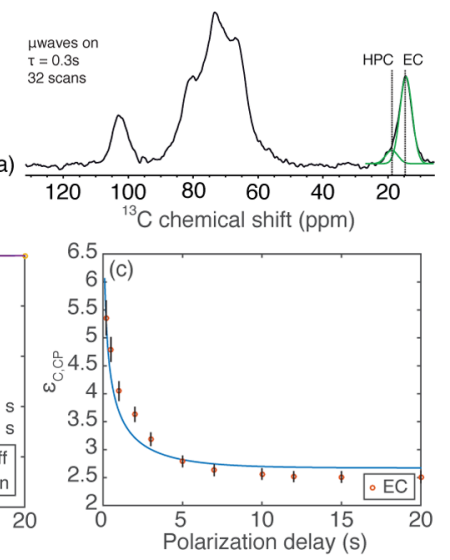

Figure 12. ${ }^{13} \mathrm{C}$ CPMAS DNP enhanced solid-state NMR spectra obtained at $105 \mathrm{~K}, B_{0}=9.4 \mathrm{~T}$, and $8 \mathrm{kHz}$ MAS for EC/HPC coated MCC pellets with $16 \mathrm{mM}$ TOTAPOL biradical in glycerol$d_{8}: \mathrm{D}_{2} \mathrm{O}: \mathrm{H}_{2} \mathrm{O}(60: 30: 10)$ solution. Because the resolution of EC and HPC methyl peaks is only partial, deconvolution was used to obtain the peak intensities (illustrated in green). (b) ${ }^{13} \mathrm{C}$ CPMAS normalized signal buildups of the EC peak. Normalization was performed using the value of the signals at steady state from a stretched exponential fit. (c) ${ }^{13} \mathrm{C}$ CP DNP enhancement of the EC peak as a function of the polarization delay. Error bars were calculated by propagation of error using the noise levels of the spectra acquired with and without microwave irradiation as the standard deviation. In panels $b$ and $c$, numerical simulations are overlapped for one-dimensional diffusion with the following parameters: $L=200 \mathrm{~nm}$ (fitted), $T_{\mathrm{B}, \text { source }}=5 \mathrm{~ms}$, $T_{1 \text {,target }}=3.5 \mathrm{~s}, D_{\text {target }}=D_{\text {source }}=2 \times 10^{-4} \mu \mathrm{m}^{2} \mathrm{~s}^{-1}, P_{\mathrm{L}}=42 \mathrm{~nm}$, and $\varepsilon_{\text {Depo }}=0.5$. The ratio of EC/HPC lengths was fixed to $70 / 30$.

above, in cases where $D$ cannot be measured experimentally independently, the result here is a measurement of the product $L \cdot D$.

\section{CONCLUSIONS}

We have shown how DNP NMR can be used in combination with models for polarization dynamics to determine the domain
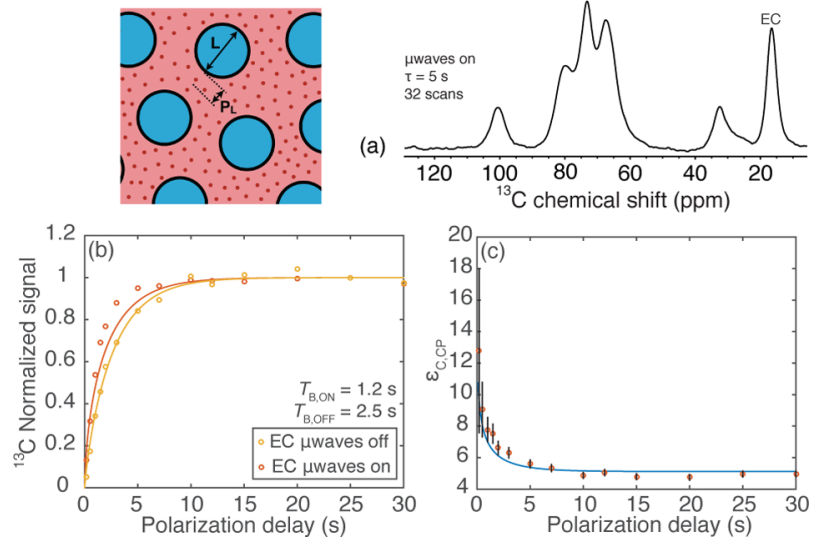

Figure 13. ${ }^{13} \mathrm{C}$ CPMAS DNP enhanced solid-state NMR spectra obtained at $95 \mathrm{~K}, B_{0}=9.4 \mathrm{~T}$ and $8 \mathrm{kHz}$ MAS of EC nanoparticles impregnated with TCE/MeOH containing bCTbK. (b) ${ }^{13} \mathrm{C}$ CPMAS normalized signal buildup of the EC peak. Normalization was performed using the value of the signals at steady of a stretched exponential fit. (c) ${ }^{13} \mathrm{C} \mathrm{CP}$ DNP enhancement of the EC peak as a function of the polarization delay. Error bars were calculated by propagation of error using the noise levels of the spectra acquired with and without microwave irradiation as the standard deviation. In panels $\mathrm{b}$ and $\mathrm{c}$, numerical simulations of a linear system are overlapped with the following parameters: $L=170 \mathrm{~nm}$ (fitted), $T_{\mathrm{B}, \text { source }}=5 \mathrm{~ms}, T_{1, \text { target }}$ $=3.7 \mathrm{~s}, P_{\mathrm{L}}=20 \mathrm{~nm}, D_{\text {target }}=D_{\text {source }}=2 \times 10^{-4} \mu \mathrm{m}^{2} \mathrm{~s}^{-1}$ and $\varepsilon_{\text {Depo }}=0.5$.

sizes in complex materials. This is achieved by measuring experimental polarization buildup curves in samples that are selectively doped with a radical containing source component. The observed polarization dynamics can be fully explained in terms of a simple model of spin diffusion between heterogeneous distributions of polarization within the sample. The DNP buildup times as well as the variation of the DNP enhancement as a function of the polarization time are found to be characteristic of the size and geometry of the micro- to nanoscale domains in the sample. We demonstrate the approach experimentally on four different systems where we successfully determine domain sizes between 200 and 20000 $\mathrm{nm}$, specifically in powdered histidine hydrochloride monohydrate, the pore lengths of mesoporous silica materials, and domain sizes in two-component polymer film coatings. Additionally, we determine experimentally that even in the apparently homogeneous frozen solutions used as polarization sources in most DNP experiments, polarization is relayed from protons near the radicals to the bulk of the solution by spin diffusion, which confirms recent predictions ${ }^{48}$ and explains the experimentally observed buildup times in these samples.

\section{ASSOCIATED CONTENT}

\section{S Supporting Information}

The Supporting Information is available free of charge on the ACS Publications website at DOI: 10.1021/acs.jpcc.7b04438.

Additional materials and methods, additional simulations (PDF)

MATLAB code used here (TXT)

Movie of polarization buildup with microwave irradiation (MOV)

Movie of polarization buildup without microwave irradiation (MOV) 


\section{AUTHOR INFORMATION}

\section{Corresponding Author}

*E-mail: lyndon.emsley@epfl.ch.

\section{ORCID $\odot$}

Mingxue Tang: 0000-0002-7282-4100

Lyndon Emsley: 0000-0003-1360-2572

\section{Notes}

The authors declare no competing financial interest.

\section{ACKNOWLEDGMENTS}

We are grateful to Matthew Conley and Christophe Copéret from ETH Zurich for providing the mesoporous silica materials. This work was supported by ERC Advanced Grant No. 320860. We are grateful to Prof. P. Tordo, Dr. O. Ouari, and Dr. G. Casano (Aix-Marseille Université, France) for providing the biradicals used in the DNP NMR experiments.

\section{REFERENCES}

(1) Wilson, J. D.; Bechtel, D. B.; Todd, T. C.; Seib, P. A. Measurement of wheat starch granule size distribution using image analysis and laser diffraction technology. Cereal Chem. 2006, 83, 259268.

(2) Hendrich, C.; Favre, L.; Ievlev, D. N.; Dobrynin, A. N.; Bras, W.; Hormann, U.; Piscopiello, E.; Van Tendeloo, G.; Lievens, P.; Temst, $\mathrm{K}$. Measurement of the size of embedded metal clusters by mass spectrometry, transmission electron microscopy, and small-angle X-ray scattering. Appl. Phys. A: Mater. Sci. Process. 2007, 86, 533-538.

(3) Amaral, S. S.; de Carvalho, J. A., Jr.; Martins Costa, M. A.; Pinheiro, C. An overview of particulate matter measurement instruments. Atmosphere 2015, 6, 1327-1345.

(4) Telkki, V. V.; Lounila, J.; Jokisaari, J. Determination of pore sizes and volumes of porous materials by Xe-129 NMR of xenon gas dissolved in a medium. J. Phys. Chem. B 2005, 109, 24343-24351.

(5) Chemmi, H.; Petit, D.; Levitz, P.; Denoyel, R.; Galarneau, A.; Korb, J.P. Noninvasive experimental evidence of the linear pore size dependence of water diffusion in nanoconfinement. J. Phys. Chem. Lett. 2016, 7, 393-398.

(6) Clauss, J.; Schmidt-Rohr, K.; Spiess, H. W. Determination of domain sizes in heterogeneous polymer by solid-state NMR. Acta Polym. 1993, 44, 1-17.

(7) Demco, D. E.; Johansson, A.; Tegenfeldt, J. Proton spin diffusion for spatial heterogeneity and morphology investigations of polymers. Solid State Nucl. Magn. Reson. 1995, 4, 13.

(8) Schmidt-Rohr, K.; Spiess, H. W. Multidimensional Solid-State NMR and Polymers; 2nd ed.; Academic Press: London, 1996.

(9) Graf, R.; Demco, D. E.; Gottwald, J.; Hafner, S.; Spiess, H. W. Dipolar couplings and internuclear distances by double-quantum nuclear magnetic resonance spectroscopy of solids. J. Chem. Phys. 1997, 106, 885-895.

(10) Goldman, M.; Shen, L. Spin-Spin Relaxation in LaF3. Phys. Rev. 1966, 144, 321.

(11) Schmidt-Rohr, K.; Clauss, J.; Blumich, B.; Spiess, H. W. Miscibility of polymer blends investigated by $\mathrm{H}-1$ spin diffusion and C13 NMR detection. Magn. Reson. Chem. 1990, 28, S3-S9.

(12) VanderHart, D. L.; McFadden, G. B. Some perspectives on the interpretation of proton NMR spin diffusion data in terms of polymer morphologies. Solid State Nucl. Magn. Reson. 1996, 7, 45-66.

(13) Schmidt-Rohr, K.; Clauss, J.; Blümich, B.; Spiess, H. W. Miscibility of polymer blends investigated by $\mathrm{H}-1$ spin diffusion and C13 NMR detection. Magn. Reson. Chem. 1990, 28, S3.

(14) Schmidt-Rohr, K.; Clauss, J.; Spiess, H. W. Correlation of structure, mobility, and morphological information in heterogeneous polymer materials by 2-dimensional wideline-separation NMR spectroscopy. Macromolecules 1992, 25, 3273-3277.

(15) Schlagnitweit, J.; Tang, M.; Baias, M.; Richardson, S.; Schantz, S.; Emsley, L. A solid-state NMR method to determine domain sizes in multi-component polymer formulations. J. Magn. Reson. 2015, 261, $43-48$.

(16) van der Wel, P. C. A.; Hu, K. N.; Lewandowski, J.; Griffin, R. G. Dynamic nuclear polarization of amyloidogenic peptide nanocrystals: GNNQQNY, a core segment of the yeast prion protein Sup35p. J. Am. Chem. Soc. 2006, 128, 10840-10846.

(17) Rossini, A. J.; Zagdoun, A.; Hegner, F. S.; Schwarzwälder, M.; Gajan, D.; Copéret, C.; Lesage, A.; Emsley, L. Dynamic nuclear polarization NMR spectroscopy of microcrystalline solids. J. Am. Chem. Soc. 2012, 134, 16899-16908.

(18) Lafon, O.; Thankamony, A. S. L.; Kobayashi, T.; Carnevale, D.; Vitzthum, V.; Slowing, II; Kandel, K.; Vezin, H.; Amoureux, J. P.; Bodenhausen, G.; et al. Mesoporous silica nanoparticles loaded with surfactant: low temperature magic angle spinning C-13 and Si-29 NMR enhanced by dynamic nuclear polarization. J. Phys. Chem. C 2013, 117, 1375-1382.

(19) Rossini, A. J.; Widdifield, C. M.; Zagdoun, A.; Lelli, M.; Schwarzwälder, M.; Copéret, C.; Lesage, A.; Emsley, L. Dynamic nuclear polarization enhanced NMR spectroscopy for pharmaceutical formulations. J. Am. Chem. Soc. 2014, 136, 2324-2334.

(20) Schlagnitweit, J.; Tang, M.; Baias, M.; Richardson, S.; Schantz, S.; Emsley, L. Nanostructure of materials determined by relayed paramagnetic relaxation enhancement. J. Am. Chem. Soc. 2015, 137, 12482-12485.

(21) Smith, A. A.; Corzilius, B.; Barnes, A. B.; Maly, T.; Griffin, R. G. Solid effect dynamic nuclear polarization and polarization pathways. J. Chem. Phys. 2012, 136, 015101.

(22) Rossini, A. J.; Zagdoun, A.; Lelli, M.; Lesage, A.; Copéret, C.; Emsley, L. Dynamic nuclear polarization surface enhanced NMR spectroscopy. Acc. Chem. Res. 2013, 46, 1942-1951.

(23) Zagdoun, A.; Casano, G.; Ouari, O.; Schwarzwälder, M.; Rossini, A. J.; Aussenac, F.; Yulikov, M.; Jeschke, G.; Copéret, C.; Lesage, A.; et al. Large molecular weight nitroxide biradicals providing efficient dynamic nuclear polarization at temperatures up to $200 \mathrm{~K}$. J. Am. Chem. Soc. 2013, 135, 12790-12797.

(24) Sauvee, C.; Rosay, M.; Casano, G.; Aussenac, F.; Weber, R. T.; Ouari, O.; Tordo, P. Highly efficient, water-soluble polarizing agents for dynamic nuclear polarization at high frequency. Angew. Chem., Int. Ed. 2013, 52, 10858-10861.

(25) Kubicki, D. J.; Casano, G.; Schwarzwalder, M.; Abel, S.; Sauvee, C.; Ganesan, K.; Yulikov, M.; Rossini, A. J.; Jeschke, G.; Coperet, C.; et al. Rational design of dinitroxide biradicals for efficient cross-effect dynamic nuclear polarization. Chemical Science 2016, 7, 550-558.

(26) Song, C. S.; Hu, K. N.; Joo, C. G.; Swager, T. M.; Griffin, R. G. TOTAPOL: A biradical polarizing agent for dynamic nuclear polarization experiments in aqueous media. J. Am. Chem. Soc. 2006, $128,11385-11390$.

(27) Peersen, O. B.; Wu, X. L.; Kustanovich, I.; Smith, S. O. Variableamplitude cross-polarization MAS NMR. J. Magn. Reson., Ser. A 1993 104, 334-339.

(28) Haze, O.; Corzilius, B.; Smith, A. A.; Griffin, R. G.; Swager, T. M. Water-soluble narrow-line radicals for dynamic nuclear polarization. J. Am. Chem. Soc. 2012, 134, 14287-14290.

(29) Bloembergen, N. On the interaction of nuclear spins in a crystalline lattice. Physica 1949, 15, 386-426.

(30) Pinon, A. C.; Rossini, A. J.; Widdifield, C. M.; Gajan, D.; Emsley, L. Polymorphs of theophylline characterized by DNP enhanced solid-state NMR. Mol. Pharmaceutics 2015, 12, 4146-4153.

(31) Bertini, I.; Luchinat, C. NMR of paramagnetic substances. Coord. Chem. Rev. 1996, 150, 1-252.

(32) Blumberg, W. E. Nuclear Spin-Lattice Relaxation Caused by Paramagnetic Impurities*. Phys. Rev. 1960, 119, 79-84.

(33) Rorschach, H. E. Nuclear Relaxation in solids by diffusion to paramagnetic impurities. Physica 1964, 30, 38-39.

(34) Tse, D.; Lowe, I. J. Nuclear spin-lattice relaxation in CaF2 crystals via paramagnetic centers. Phys. Rev. 1968, 166, 292-302.

(35) Brownstein, K. R.; Tarr, C. E. Phys. Rev. A: At., Mol., Opt. Phys. $1979,19,2446$ 
(36) Allen, S. G.; Stephenson, P. C. L.; Strange, J. H. Morphology of porous media studied by nuclear magnetic resonance. J. Chem. Phys. 1997, 106, 7802 .

(37) Bertini, I.; Luchinat, C.; Parigi, G. Solution NMR of Paramagnetic Molecules: Applications to Metallobiomolecules and Models; Elsevier: Amsterdam, 2001.

(38) Rabbani, S. R.; Mendonça, C.; Mamani, J. B.; Rodriguez, H. C. Analysis of nuclear relaxation in granular systems. Braz. J. Phys. 2006, $36,28$.

(39) Pintacuda, G.; John, M.; Su, X. C.; Otting, G. NMR Structure determination of protein-ligand complexes by lanthanide labeling. Acc. Chem. Res. 2007, 40, 206.

(40) Lubach, J. W.; Xu, D.; Segmuller, B. E.; Munson, E. J. Investigation of the effects of pharmaceutical processing upon solidstate NMR relaxation times and implications to solid-state formulation stability. J. Pharm. Sci. 2007, 96, 777.

(41) Dupre, N.; Martin, J. F.; Guyomard, D.; Yamada, A.; Kanno, R Detection of surface layers using (7)Li MAS NMR. J. Mater. Chem. 2008, 18, 4266.

(42) Henrichs, P. M.; Linder, M.; Hewitt, J. M. Dynamics of the C-13 spin-exchange process in solids - a theoretical and experimental study. J. Chem. Phys. 1986, 85, 7077-7086.

(43) Suter, D.; Ernst, R. R. Spin diffusion in resolved sold-state NMR spectra. Phys. Rev. B: Condens. Matter Mater. Phys. 1985, 32, 56085627.

(44) Halse, M. E.; Zagdoun, A.; Dumez, J.-N.; Emsley, L. Macroscopic nuclear spin diffusion constants of rotating polycrystalline solids from first-principles simulation. J. Magn. Reson. 2015, 254, $48-55$.

(45) Dumez, J. N.; Halse, M. E.; Butler, M. C.; Emsley, L. A firstprinciples description of proton-driven spin diffusion. Phys. Chem. Chem. Phys. 2012, 14, 86-89.

(46) Karabanov, A.; Wisniewski, D.; Lesanovsky, I.; Koeckenberger, W. Dynamic nuclear polarization as kinetically constrained diffusion. Phys. Rev. Lett. 2015, 115, 020404.

(47) Kubo, A.; McDowell, C. A. Spectral spin diffusion in polycrystalline solids under magic angle spinning. J. Chem. Soc., Faraday Trans. 1 1988, 84, 3713-3730.

(48) Mentink-Vigier, F.; Vega, S.; De Paëpe, G. Fast and accurate MAS-DNP simulations of large spin ensembles. Phys. Chem. Chem. Phys. 2017, 19, 3506.

(49) Mentink-Vigier, F.; Paul, S.; Lee, D.; Feintuch, A.; Hediger, S.; Vega, S.; De Paepe, G. Nuclear depolarization and absolute sensitivity in magic-angle spinning cross effect dynamic nuclear polarization. Phys. Chem. Chem. Phys. 2015, 17, 21824-21836.

(50) Thurber, K. R.; Tycko, R. Perturbation of nuclear spin polarizations in solid state NMR of nitroxide-doped samples by magic-angle spinning without microwaves. J. Chem. Phys. 2014, 140, 184201.

(51) Paul, A.; Laurila, T.; Vuorinen, V.; Divinski, S. V. Thermodynamics, Diffusion and the Kirkendall Effect in Solids; Springer: Cham, Switzerland, 2014.

(52) Fick, A. Ueber diffusion. Ann. Phys. 1855, 170, 59-86.

(53) Girifalco, L. A. Statistical Mechanics of Solids; Oxford University Press: New York, 2000.

(54) Kubicki, D. J.; Rossini, A. J.; Purea, A.; Zagdoun, A.; Ouari, O.; Tordo, P.; Engelke, F.; Lesage, A.; Emsley, L. Amplifying dynamic nuclear polarization of frozensolutions by incorporating dielectric particles. J. Am. Chem. Soc. 2014, 136, 15711-15718.

(55) Zagdoun, A.; Rossini, A. J.; Gajan, D.; Bourdolle, A.; Ouari, O.; Rosay, M.; Maas, W. E.; Tordo, P.; Lelli, M.; et al. Nonaqueous solvents for DNP spectroscopy. Chem. Commun. 2012, 48, 654-656.

(56) Hu, K. N.; Yu, H. H.; Swager, T. M.; Griffin, R. G. Dynamic nuclear polarization with biradicals. J. Am. Chem. Soc. 2004, 126, 10844-10845.

(57) Lange, S.; Linden, A. H.; Akbey, U.; Franks, W. T.; Loening, N. M.; van Rossum, B.-J.; Oschkinat, $H$. The effect of biradical concentration on the performance of DNP-MAS-NMR. J. Magn. Reson. 2012, 216, 209-212.
(58) Ji, X.; Bornet, A.; Vuichoud, B.; Milani, J.; Gajan, D.; Rossini, A. J.; Emsley, L.; Bodenhausen, G.; Jannin, S. Transportable hyperpolarized metabolites. Nat. Commun. 2017, 8, 13975.

(59) Rossini, A. J.; Zagdoun, A.; Lelli, M.; Canivet, J.; Aguado, S.; Ouari, O.; Tordo, P.; Rosay, M.; Maas, W. E.; et al. Dynamic nuclear polarization enhanced solid-state NMR spectroscopy of functionalized metal-organic frameworks. Angew. Chem., Int. Ed. 2012, 51, 123-127.

(60) Pump, E.; Viger-Gravel, J.; Abou-Hamad, E.; Samantaray, M. K.; Hamzaoui, B.; Gurinov, A.; Anjum, D. H.; Gajan, D.; Lesage, A.; Bendjeriou-Sedjerari, A.; et al. Reactive surface organometallic complexes observed using dynamic nuclear polarization surface enhanced NMR spectroscopy. Chemical Science 2017, 8, 284-290.

(61) Crank, J. The Mathematics of Diffusion; Oxford University Press: Oxford, U.K., 1979.

(62) Weibull, W. A Statistical distribution function of wide applicability. J. Appl. Mech. 1951, 18, 293-297.

(63) Roos, M.; Micke, P.; Saalwachter, K.; Hempel, G. Moderate MAS enhances local H-1 spin exchange and spin diffusion. J. Magn. Reson. 2015, 260, 28-37.

(64) Wilhelm, M.; Feng, H.; Tracht, U.; Spiess, H. W. 2D CP/MAS $\mathrm{C}-13$ isotropic chemical shift correlation established by $\mathrm{H}-1$ spin diffusion. J. Magn. Reson. 1998, 134, 255-260. 


\title{
Measuring Nano to Micro Structures from Relayed DNP NMR
}

\author{
Arthur C. Pinon, ${ }^{1}$ Judith Schlagnitweit, ${ }^{2,4}$ Pierrick Berruyer, ${ }^{4}$ Aaron J. Rossini, ${ }^{1,5}$ Moreno
} Lelli, ${ }^{6}$ Etienne Socie, ${ }^{1}$ Mingxue Tang, ${ }^{7}$ Tran Pham, ${ }^{3}$ Anne Lesage, ${ }^{4}$ Staffan Schantz, ${ }^{8}$ and Lyndon Emsley. ${ }^{1}$

\footnotetext{
${ }^{1}$ Institut des Sciences et Ingénierie Chimiques, Ecole Polytechnique Fédérale de Lausanne (EPFL), CH-1015 Lausanne, Switzerland.

${ }^{2}$ Karolinska Institutet, Stockholm, Sweden

${ }^{3}$ GSK Medicines Research Centre, Stevenage, SG1-2NY, United Kingdom.

${ }^{4}$ Centre de Résonance Magnétique Nucléaire (RMN) à Très Hauts Champs, Institut des Sciences Analytiques (CNRS/ENS de Lyon/ UCB Lyon 1), Université de Lyon, 69100 Villeurbanne, France.

${ }^{5}$ Department of Chemistry, Iowa State University, Ames, IA 50011-3111, USA.

${ }^{6}$ Department of Chemistry, University of Florence, Center for Magnetic Resonance, 50019 Sesto Fiorentino (FI), Italy

${ }^{7}$ National High Magnetic Field Laboratory, 1800 East Paul Dirac Drive, Tallahassee, FL-32310, USA.

${ }^{8}$ Pharmaceutical Development, AstraZeneca R\&D Mölndal, SE-431 83 Mölndal, Sweden
}

\section{Supporting information}

Table of Contents

\begin{tabular}{|l|l|}
\hline Experimental details & $\mathrm{S} 2$ \\
\hline Hyperbolic secant function details and parameters & $\mathrm{S} 2$ \\
\hline Figure S1. Target enhancement as a function of the position & $\mathrm{S} 3$ \\
\hline Figure S2. Influence of the spinning rate of the sample & $\mathrm{S} 4$ \\
\hline Figure S3. Influence of the border thickness & $\mathrm{S} 4$ \\
\hline $\begin{array}{l}\text { Figure S4. Influence of the fraction of the observable signal for a thick border } \\
\text { Figure S5. Influence of the fraction of the observable signal for a thin border }\end{array}$ & $\mathrm{S} 5$ \\
\hline Figure S6. Influence of the length of the source & $\mathrm{S} 6$ \\
\hline Figure S7. Influence of the source enhancement & $\mathrm{S} 6$ \\
\hline Figure S8. Influence of $T_{1, \text { target }}$ & $\mathrm{S} 7$ \\
\hline Figure S9. Influence of $T_{1, \text { source }}$ & $\mathrm{S} 7$ \\
\hline Figure S10-11-12. Influence of $T_{1, \text { target }}$ on the steady state enhancement & $\mathrm{S} 8$ \\
\hline $\begin{array}{l}\text { Figure S13. } \beta \text { factor of the stretched exponential fitting functions as a function of the length } \\
\text { of the target }\end{array}$ & $\mathrm{S} 10$ \\
\hline
\end{tabular}




\begin{tabular}{|l|l|}
\hline Figure S14. SEM image of the ground histidine powder & S11 \\
\hline Figure S15. Size distribution of EC lengths for the aquacoat study & S11 \\
\hline Figure S16. Unground Histidine Powder polarized by Relayed DNP & S12 \\
\hline Figure S17. Why the enhancement increases at early times & S13 \\
\hline Figure S18. Definition of characteristic diffusion length and characteristic diffusion time & S14 \\
\hline $\begin{array}{l}\text { Figure S19-20. Diffusion rate profile as a function of position, close to the electron location, } \\
\text { together with the corresponding build-up }\end{array}$ & S20 \\
\hline
\end{tabular}

\section{Experimental details}

\section{$\underline{\text { Elemental Analysis }}$}

Elemental analysis was carried out by Mikroanalytisches Labor Pascher.

\section{Synthesis of Material Ph-Im-OH}

$\mathrm{Si}\left(\mathrm{CD}_{3}\right)_{3} \mathrm{Cl}$ was purchased from Cortecnet and used as received. Triethylamine was purchased from Fluka and distilled from $\mathrm{CaH}_{2}$ before use. In an Ar-filled glovebox, a $100 \mathrm{~mL}$ round-bottomed flask equipped with a stir bar was charged with $1 \mathrm{~g}$ of the precursor, sealed with a rubber septum and electrical tape, and brought into a fume hood. A needle connected to a flow of Ar was attached to the vessel and, via syringe, $20 \mathrm{~mL}$ of toluene was added followed by $0.31 \mathrm{~mL}(0.35 \mathrm{~g}, 2.4 \mathrm{mmol})$ of 1-phenylimidazole. The rubber septum was quickly exchanged for a reflux condenser capped with a rubber septum. The reaction mixture was then heated to reflux and allowed to stir for $18 \mathrm{~h}$ while being protected from light with aluminum foil. After allowing the reaction mixture to cool to room temperature, the resulting pale yellow solid was collected on a porosity 3 fritted funnel and was washed with dichloromethane ( 3 x 30 $\mathrm{mL}$ ). The solid was then transferred to a $100 \mathrm{~mL}$ round-bottomed flask containing a stirring mixture of pyridine, deionized water, and $2 \mathrm{M}$ aqueous $\mathrm{HCl}(18: 18: 6 \mathrm{~mL}$, respectively), and the reaction vessel was capped with a rubber septum containing a vent needle. The reaction mixture was heated to $50{ }^{\circ} \mathrm{C}$ and allowed to stir for $20 \mathrm{~h}$. After allowing the reaction mixture to cool to room temperature, the resulting pale yellow solid was collected on a porosity 3 fritted funnel and was washed with deionized $\mathrm{H}_{2} \mathrm{O}(3 \mathrm{x}$ $30 \mathrm{~mL})$, acetone $(3 \times 30 \mathrm{~mL})$, and diethyl either $(3 \times 30 \mathrm{~mL})$. The solid was then dried for 12 hours under a vacuum of less than $10^{-4}$ Torr at $135^{\circ} \mathrm{C}$ using a temperature ramp of $1{ }^{\circ} \mathrm{C} / \mathrm{min}$ from room temperature $\left(\sim 23{ }^{\circ} \mathrm{C}\right)$ and brought into an Ar-filled glovebox. Following drying, $0.80 \mathrm{~g}$ of an off-white solid was obtained. Elemental analyswas: $\mathrm{C}=6.3 \%_{\mathrm{wt}} ; \mathrm{H}=1.0 \%_{\mathrm{wt}} ; \mathrm{N}=1.2 \%_{\mathrm{wt}}$. C/N ratio obtained: 5.9 (expected $6.0)$.

\section{Synthesis of Material Ph-Im-OSi $\left(\mathrm{CD}_{\underline{3}}\right)_{3}$}

In an Ar-filled glovebox, a $25 \mathrm{~mL}$ round-bottomed flask equipped with a stir bar is charged with $0.60 \mathrm{~g}$ (0.29 mmol) of Ph-Im-OH, sealed with a rubber septum and electrical tape, and brought into a fume hood. A needle connected to a flow of Ar is attached to the vessel and toluene $(10 \mathrm{~mL})$, triethylamine $(2.5 \mathrm{~mL}, 18 \mathrm{mmol})$, and $\mathrm{Si}\left(\mathrm{CD}_{3}\right)_{3} \mathrm{Cl}(1.2 \mathrm{~mL}, 8.7 \mathrm{mmol})$ are added via syringe in that order. The reaction is then allowed to stir at room temperature $\left(\sim 23^{\circ} \mathrm{C}\right)$ for $18 \mathrm{~h}$. The reaction mixture is filtered through a porosity 3 fritted funnel and the resulting solid is washed with deionized water $(2 \times 50 \mathrm{~mL})$ and acetone $(2 \times 50 \mathrm{~mL})$. The white powder is then dried for 12 hours under a vacuum of less than $10^{-4}$ Torr at 135 ${ }^{\circ} \mathrm{C}$ using a temperature ramp of $1{ }^{\circ} \mathrm{C} / \mathrm{min}$ from room temperature $\left(\sim 23{ }^{\circ} \mathrm{C}\right)$ and brought into an Ar-filled 
glovebox. Following drying, $0.62 \mathrm{~g}$ of an off- white powder was obtained. Elemental analysis: $\mathrm{C}=$ $11.4 \%_{\mathrm{wt}} ; \mathrm{H}=0.6 \%_{\mathrm{wt}} ; \mathrm{N}=1.0 \%_{\mathrm{wt}}$.

\section{Hyperbolic secant functions:}

For a 1 dimensional system with 2 sources:

$$
\begin{gathered}
T_{1}(x)=T_{1, \text { source }}+\frac{T_{1, \text { target }}-T_{1, \text { source }}}{2}\left[\tanh \left(p\left(x-P_{L}\right)\right)+\tanh \left(p\left(L-x-P_{L}\right)\right]\right. \\
P_{0, \text { on }}(x)=\varepsilon_{0}+\frac{1-\varepsilon_{0}}{2}\left[\tanh \left(p\left(x-P_{L}\right)\right)+\tanh \left(p\left(L-x-P_{L}\right)\right]\right. \\
D(x)=D_{\text {source }}+\frac{D_{\text {target }}-D_{\text {source }}}{2}\left[\tanh \left(p\left(x-P_{L}\right)\right)+\tanh \left(p\left(L-x-P_{L}\right)\right]\right.
\end{gathered}
$$

For a 3 dimensional system with 1 source:

$$
\begin{gathered}
T_{1}(x)=\frac{T_{1, \text { target }}+T_{1, \text { source }}}{2}+\frac{T_{1, \text { target }}-T_{1, \text { source }}}{2}\left[\tanh \left(p\left(L-x-P_{L}\right)\right]\right. \\
P_{0, \text { on }}(x)=\frac{\varepsilon_{0}+1}{2}+\frac{1-\varepsilon_{0}}{2}\left[\tanh \left(p\left(L-x-P_{L}\right)\right]\right. \\
D(x)=\frac{D_{\text {source }}+D_{\text {target }}}{2}+\frac{D_{\text {target }}-D_{\text {source }}}{2}\left[\tanh \left(p\left(L-x-P_{L}\right)\right]\right.
\end{gathered}
$$

where $x$ is the position in $\mu \mathrm{m}, \mathrm{p}$ is the slope of the inflexion point of the hyperbolic secant function, which defines the thickness of the border, $T_{1}$ the relaxation time in seconds, $D$ the diffusion coefficient in $\mu \mathrm{m}^{2} . \mathrm{s}^{-1}, \varepsilon_{0}$ the local equilibrium enhancement of the source, $L$ the length of the target, and $P_{\mathrm{L}}$ the length of the source, both in $\mu \mathrm{m}$.

The $1 / \mathrm{r}^{6}$ function presented in the main text are described as:

$$
A(x)=\frac{1}{\frac{1}{A_{\min }}+\frac{A_{\min }-A_{\max }}{A_{\min } A_{\max }}\left(\frac{210^{-3}}{210^{-3}-L-x}\right)^{6}}
$$

where $A_{\min }$ and $A_{\max }$ are the minimal and maximal values of $A$, and $L$ the length of the target.

In the next simulations, a quenching factor was taken into account in the source. We define $\theta_{\min }$ as the fraction of observed signal.

\section{Enhancement of the target as a function of position:}




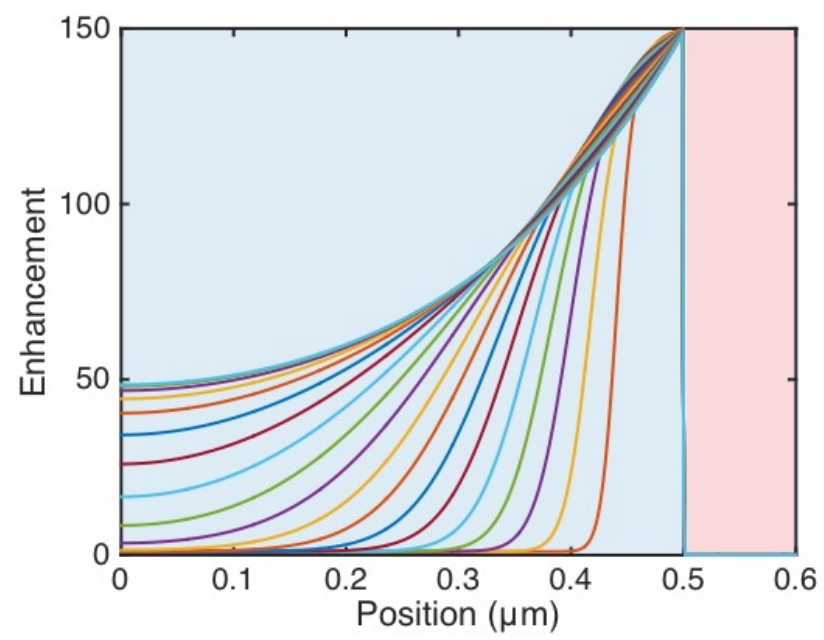

Figure S1. Simulations of the enhancement as a function of the position in a system with $\mu$ wave irradiations, for different times from $\mathrm{t}=0$ to $200 \mathrm{~s}$ (steady state reached) with exponential time steps, in a 3D system. The source is shown in a red background, whereas the target is shown in a blue background. In the simulation, $T_{1 \text {, source }}=5 \mathrm{~ms}, T_{1, \text { target }}=30 \mathrm{~s}, \varepsilon_{0}=150, D_{\text {target }}=1.10^{-3} \mu \mathrm{m}^{2} \mathrm{~s}^{-1}, P_{\mathrm{L}}=0.1 \mu \mathrm{m}$, and $\theta_{\min }=0$ in the source. The border thickness was $2 \mathrm{~nm}$.

\section{Influence of the spinning rate.}
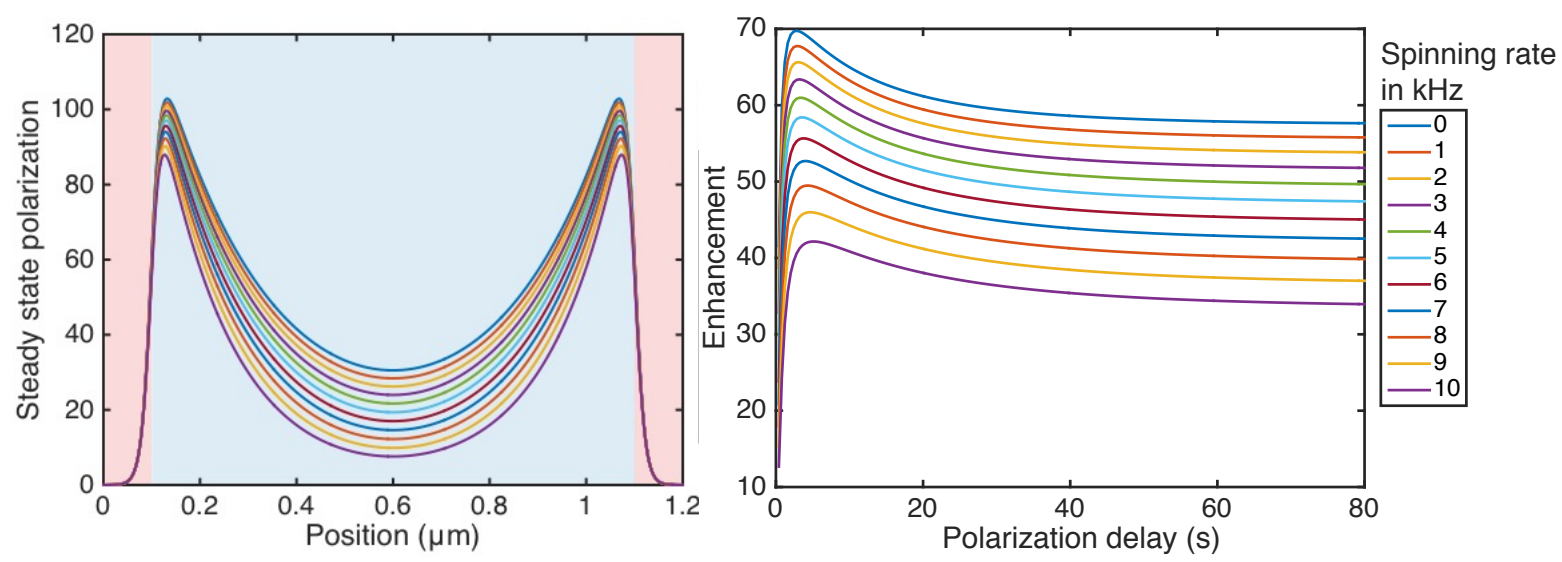

Figure S2. Left: Simulated polarization at steady state in a 1D system as a function of the position, for different spinning rates. Right: Simulated target enhancement as a function of the polarization delay, for different spinning rates. In all simulations, $\mathrm{L}=1.2 \mu \mathrm{m}, T_{1, \text { source }}=5 \mathrm{~ms}, T_{1, \text { target }}=30 \mathrm{~s}, \varepsilon_{0}=150, P_{\mathrm{L}}=0.1$ $\mu \mathrm{m}, \theta_{\min }=0$ in the source, and the border thickness was $200 \mathrm{~nm}$. The spinning rate was taken to be inversely proportional to the diffusion coefficient, as described in ref ${ }^{1}$.

\section{Influence of the border thickness.}

The slope of the inflexion point (parameter $p$ ) of the hyperbolic secant function defines the thickness of the border between the source and the target. 

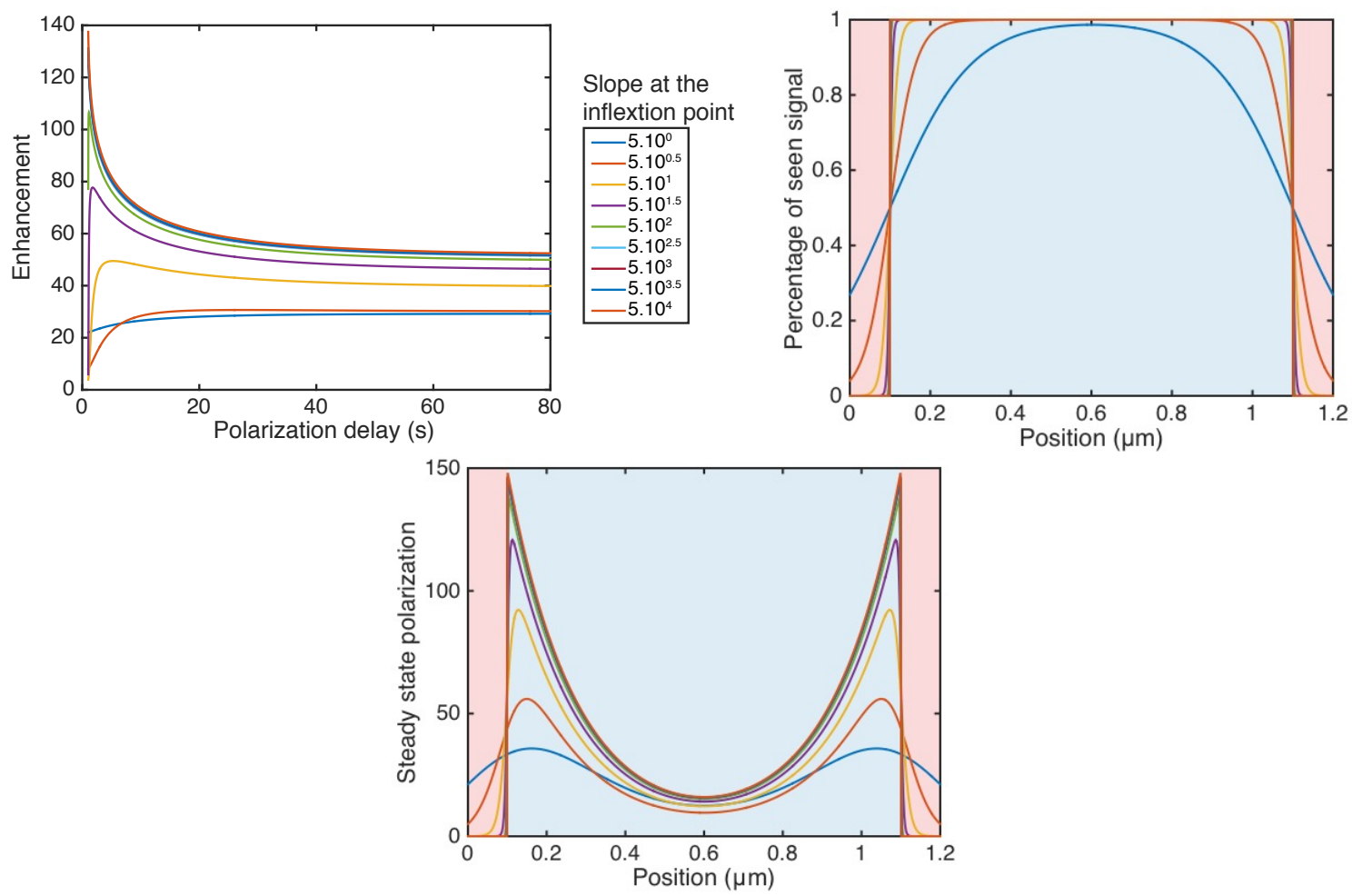

Figure S3. Top left graph shows the simulated target enhancement of a 1D system as a function of the polarization delay for different slopes at the inflexion point of the hyperbolic secant function (i.e. for different border thicknesses). Top right graph shows the simulated percentage of observable signal of a $1 \mathrm{D}$ system as a function of the position for different border thicknesses. Bottom graph shows the simulated $\mu$ wave on polarization at steady state of a $1 \mathrm{D}$ system as a function of position for different border thicknesses. In all simulations, $\mathrm{L}=1.2 \mu \mathrm{m}, T_{1, \text { source }}=5 \mathrm{~ms}, T_{1, \text { target }}=30 \mathrm{~s}, \varepsilon_{0}=150, D_{\text {target }}=D_{\text {source }}=1.10^{-3}$ $\mu \mathrm{m}^{2} \mathrm{~s}^{-1}, P_{\mathrm{L}}=0.1 \mu \mathrm{m}, \theta_{\min }=0$.

\section{Influence of the fraction of observable signal in the source for a thick border.}
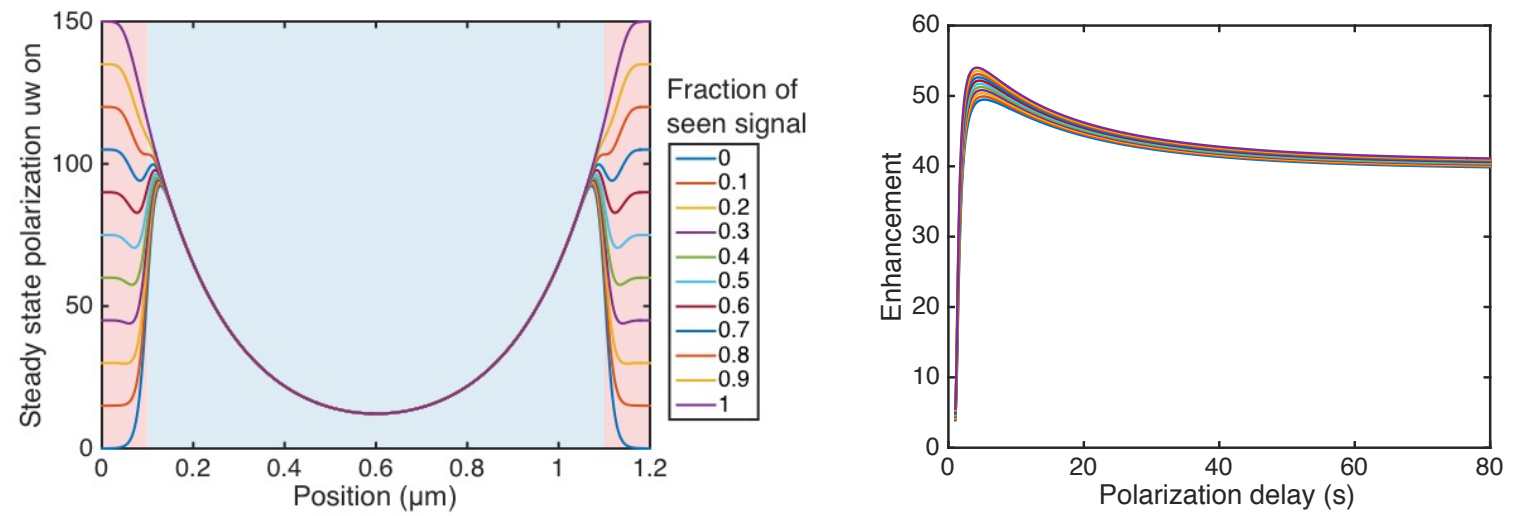

Figure S4. Left graph shows the simulated $\mu$ wave on polarization at steady state of a $1 \mathrm{D}$ system as a function of position for different fractions of observable signal in the source. Right graph shows the simulated target enhancement of a 1D system as a function of the polarization delay for different fractions of observable signal in the source. In all simulations, $\mathrm{L}=1.2 \mu \mathrm{m}, T_{1, \text { source }}=5 \mathrm{~ms}, T_{1, \text { target }}=30 \mathrm{~s}, \varepsilon_{0}=$ $150, D_{\text {target }}=D_{\text {source }}=1.10^{-3} \mu \mathrm{m}^{2} \mathrm{~s}^{-1}, P_{\mathrm{L}}=0.1 \mu \mathrm{m}$, and the border thickness was $\underline{200 \mathrm{~nm}}$. 
Influence of the fraction of observable signal in the source for a thin border.
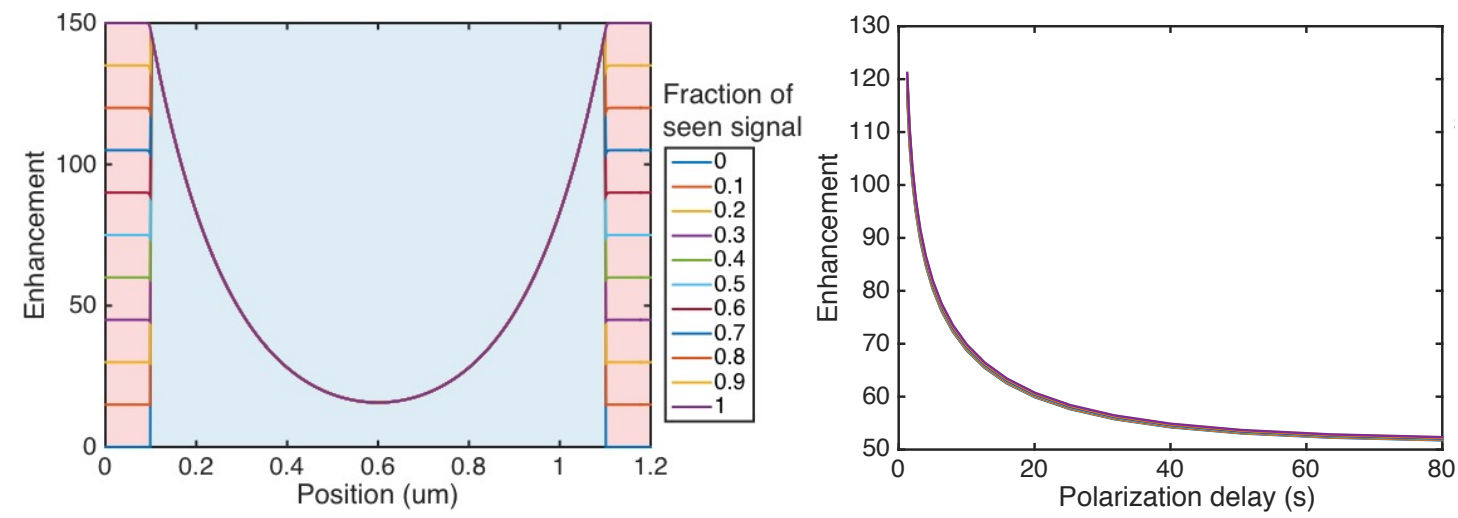

Figure S5. Left graph shows the simulated $\mu$ wave on polarization at steady state of a 1D system as a function of position for different fractions of observable signal in the source. Right graph shows the target enhancement of a 1D system as a function of the recycle delay for different fractions of observable signal in the source. In all simulations, $\mathrm{L}=1.2 \mu \mathrm{m}, T_{1, \text { source }}=5 \mathrm{~ms}, T_{1, \text { target }}=30 \mathrm{~s}, \varepsilon_{0}=150, D_{\text {target }}=D_{\text {source }}$ $=1.10^{-3} \mu \mathrm{m}^{2} \mathrm{~s}^{-1}, P_{\mathrm{L}}=0.1 \mu \mathrm{m}$, and the border thickness was $\underline{2 \mathrm{~nm}}$.

\section{Influence of the length of the source.}
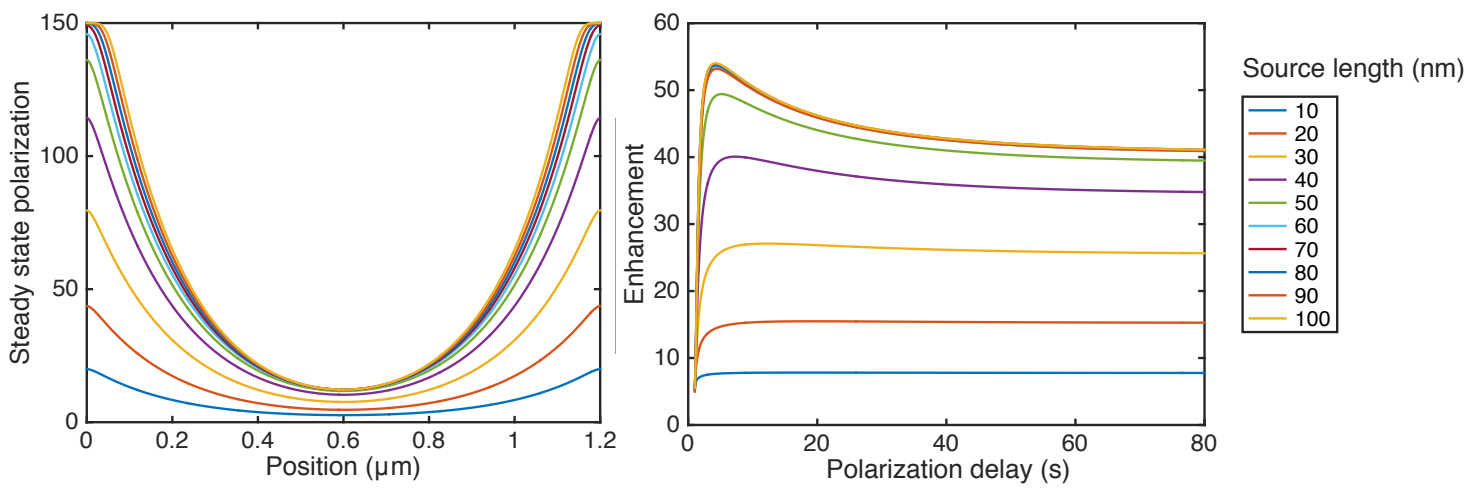

Figure S6. Left graph shows the simulated $\mu$ wave on polarization at steady state of a 1D system as a function of position for different source lengths. Right graph shows the target enhancement of a 1D system as a function of the polarization delay for different source lengths. In all simulations, $\mathrm{L}=1.2 \mu \mathrm{m}$, $T_{1, \text { source }}=5 \mathrm{~ms}, T_{1, \text { target }}=30 \mathrm{~s}, D_{\text {target }}=D_{\text {source }}=1.10^{-3} \mu \mathrm{m}^{2} \mathrm{~s}^{-1}, P_{\mathrm{L}}=0.1 \mu \mathrm{m}, \varepsilon_{0}=150, \theta_{\min }=0$, and the border thickness was $200 \mathrm{~nm}$.

\section{Influence of the source enhancement.}



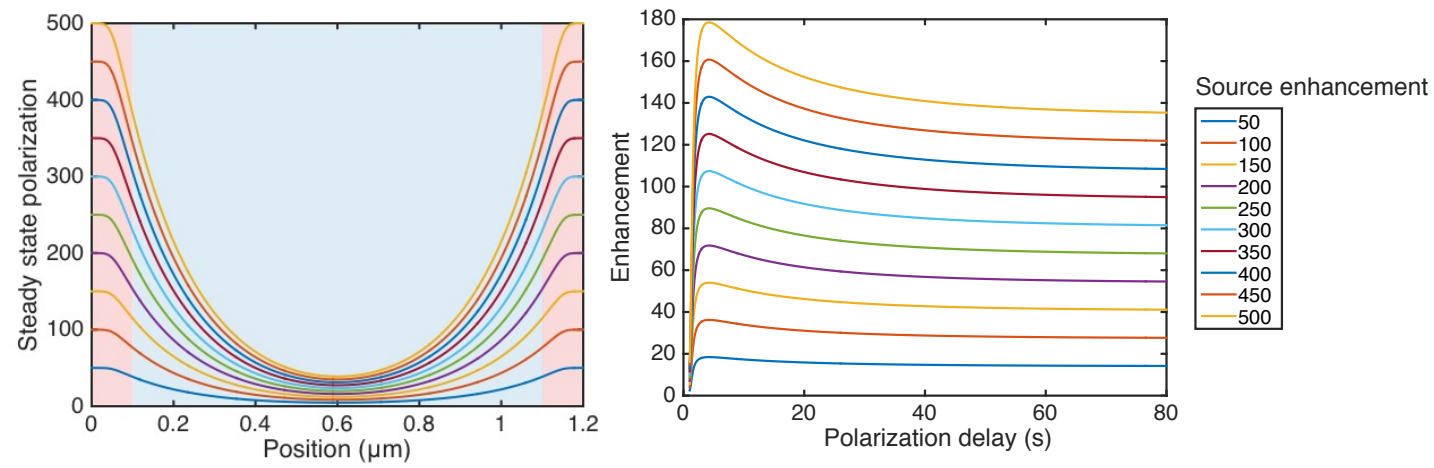

Figure S7. Left graph shows the $\mu$ wave on polarization at steady state of a 1D system as a function of position for different source enhancements. Right graph shows the target enhancement of a 1D system as a function of the recycle delay for different source enhancements. In all simulations, $\mathrm{L}=1.2 \mu \mathrm{m}$, $T_{1, \text { source }}=5 \mathrm{~ms}, T_{1 \text {,target }}=30 \mathrm{~s}, \varepsilon_{0}=150, D_{\text {target }}=D_{\text {source }}=1.10^{-3} \mu \mathrm{m}^{2} \mathrm{~s}^{-1}, P_{\mathrm{L}}=0.1 \mu \mathrm{m}, \theta_{\text {min }}=0$, and the border thickness was $200 \mathrm{~nm}$.

\section{Influence of $T_{1, \text { target }}$.}
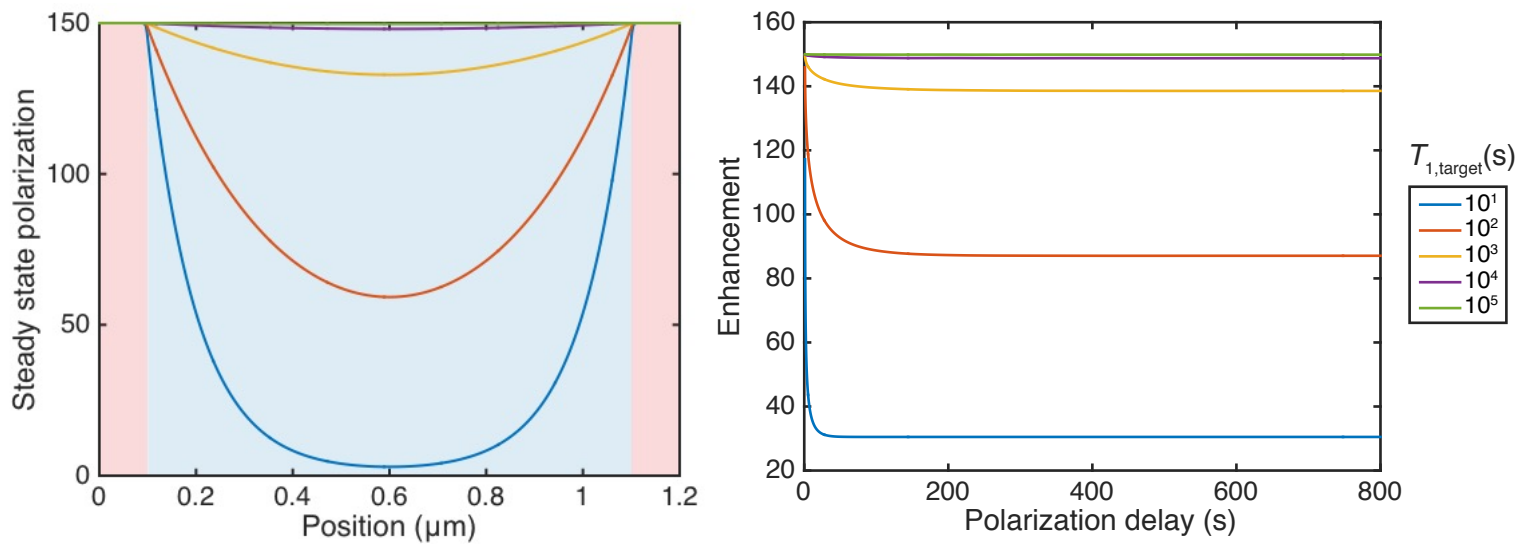

Figure S8. Left graph shows the microwave on polarization at steady state of a 1D system as a function of position for different longitudinal relaxation times in the target. Right graph shows the target enhancement of a 1D system as a function of the polarization delay for different longitudinal relaxation times in the target. In all simulations, $\mathrm{L}=1.2 \mu \mathrm{m}, T_{1 \text {, source }}=5 \mathrm{~ms}, \varepsilon_{0}=150, D_{\text {target }}=D_{\text {source }}=1.10^{-3} \mu \mathrm{m}^{2} \mathrm{~s}^{-1}$, $P_{\mathrm{L}}=0.1 \mu \mathrm{m}, \theta_{\text {min }}=0$, and the border thickness was $200 \mathrm{~nm}$.

\section{Influence of $T_{1, \text { source }}$.}
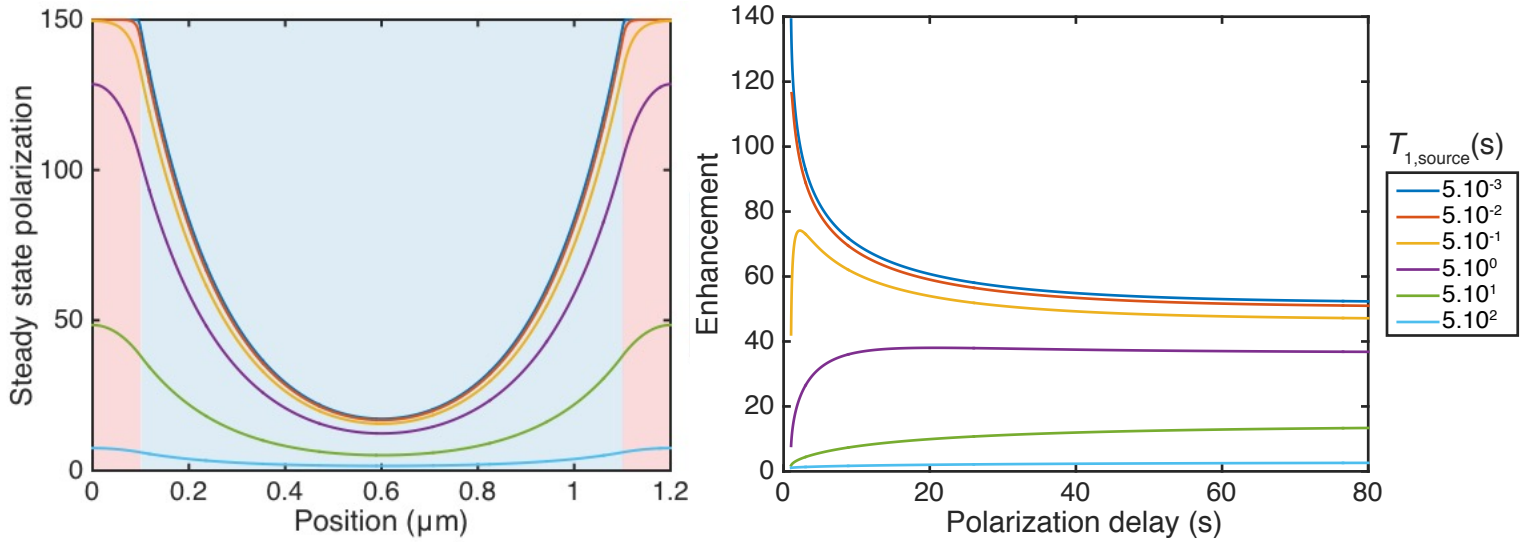
Figure S9. Left graph shows the $\mu$ wave on polarization at steady state of a 1D system as a function of position for different longitudinal relaxation times in the source. Left graph shows the target enhancement of a $1 \mathrm{D}$ system as a function of the polarization delay for different longitudinal relaxation times in the source. In all simulations, $\mathrm{L}=1.2 \mu \mathrm{m}, T_{1, \text { target }}=30 \mathrm{~s}, \varepsilon_{0}=150, D_{\text {target }}=D_{\text {source }}=1.10^{-3} \mu \mathrm{m}^{2} \mathrm{~s}^{-1}, P_{\mathrm{L}}=0.1$ $\mu \mathrm{m}, \theta_{\min }=0$, and the border thickness was $200 \mathrm{~nm}$.

\section{Influence of $T_{1, \text { target }}$ on the enhancement at steady state.}

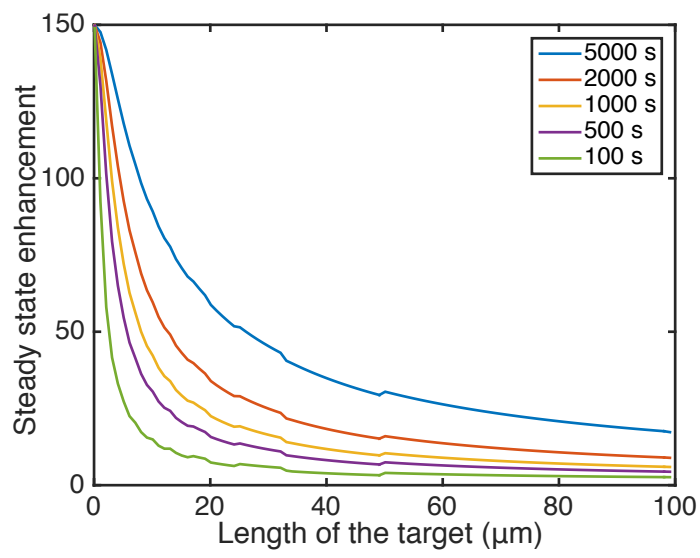

Figure S10. Simulated enhancement at steady state of a 3D system as a function of position for different longitudinal relaxation times in the target. In all simulations, $T_{1, \text { source }}=5 \mathrm{~ms}, \varepsilon_{0}=150, D_{\text {target }}=D_{\text {source }}=$ $1.10^{-3} \mu \mathrm{m}^{2} \mathrm{~s}^{-1}, P_{\mathrm{L}}=0.1 \mu \mathrm{m}, \varepsilon_{0}=150, \theta_{\text {min }}=0$, and the border thickness was $2 \mathrm{~nm} . \mathrm{N}$

Little jumps in the curves correspond to numerical errors.

\section{Modeled depolarization factor vs. literature depolarization factor:}

In the following, we will only be concerned about the source.

The depolarization factor is described in the literature for bulk sample as: ${ }^{2}$

$$
\varepsilon_{\text {Depo,lit }}=\frac{S_{\text {off }}(t, v>0)}{S_{\text {off }}(t, v=0)}
$$

As described in the main text, a bulk sample doesn't contain any heterogeneity of radical distribution, thus no heterogeneity of polarization. There is no substantive spin diffusion in such system. The observed build-up time is thus following $T_{\mathrm{B}, \mathrm{source}}$. The intrinsic longitudinal relaxation time doesn't depend on the spinning rate, so neither the build-up time. Thus, the signal build-up follows:

$$
S_{\text {off }}(t, v>0)=\varepsilon_{\text {Depo,pol }}(v)\left(1-\mathrm{e}^{-t / T_{B, \text { source }}}\right)
$$

where we define $\varepsilon_{\text {Depo,pol }}(v)$ as

$$
\varepsilon_{\text {Depo,pol }}(v)=\frac{P_{0, \text { off }}(v>0)}{P_{0, \text { off }}(v=0)}
$$

and

$$
S_{\text {off }}(t, v=0)=1-\mathrm{e}^{-t / T_{1, \text { source }}}
$$


The depolarization factor in this case becomes:

$$
\varepsilon_{\text {Depo,lit }}=\frac{\varepsilon_{\text {Depo,pol }}(v)\left(1-\mathrm{e}^{-t / T_{B, \text { source }}}\right)}{1-\mathrm{e}^{-t / T_{B, \text { source }}}}=\varepsilon_{\text {Depo,pol }}(v)
$$

In other words:

$$
\frac{S_{\text {off }}(t, v>0)}{S_{\text {off }}(t, v=0)}=\frac{P_{0, \text { off }}(v>0)}{P_{0, \text { off }}(v=0)}
$$

It means that, in a homogeneous system, when we can define the depolarization factor as the ratio of local equilibrium polarization factor instead of the ratio of signals. The main consequence is that we can assert that the depolarization factor doesn't depend on time.

For a heterogeneous system, the build-up is no longer mono-exponential since spin diffusion occurs. The depolarization factor theoretically depends on time, and be different from a simple ratio of local equilibrium polarizations as for a homogeneous system like a bulk a sample. We thus calculated the depolarization factor as a function of time for a system with typical values of diffusion coefficient and relaxation times: $D_{\text {source }}=D_{\text {target }}=1.10^{-3} \mu \mathrm{m}^{2} \mathrm{~s}^{-1}, T_{1 \text {,source }}=3 \mathrm{~s}$ and $T_{1, \text { target }}=30 \mathrm{~s}$ as shown in Figure $\mathrm{S} 11$. We see that the actual depolarization factor plotted on the right graph, which is the ratio of the signals, is quite close to the ratio of the local equilibrium polarizations (which is 0.5 ). In the main text, we assume that the ratio of signals is equal to the ratio of local equilibrium polarizations, as for a homogeneous system.
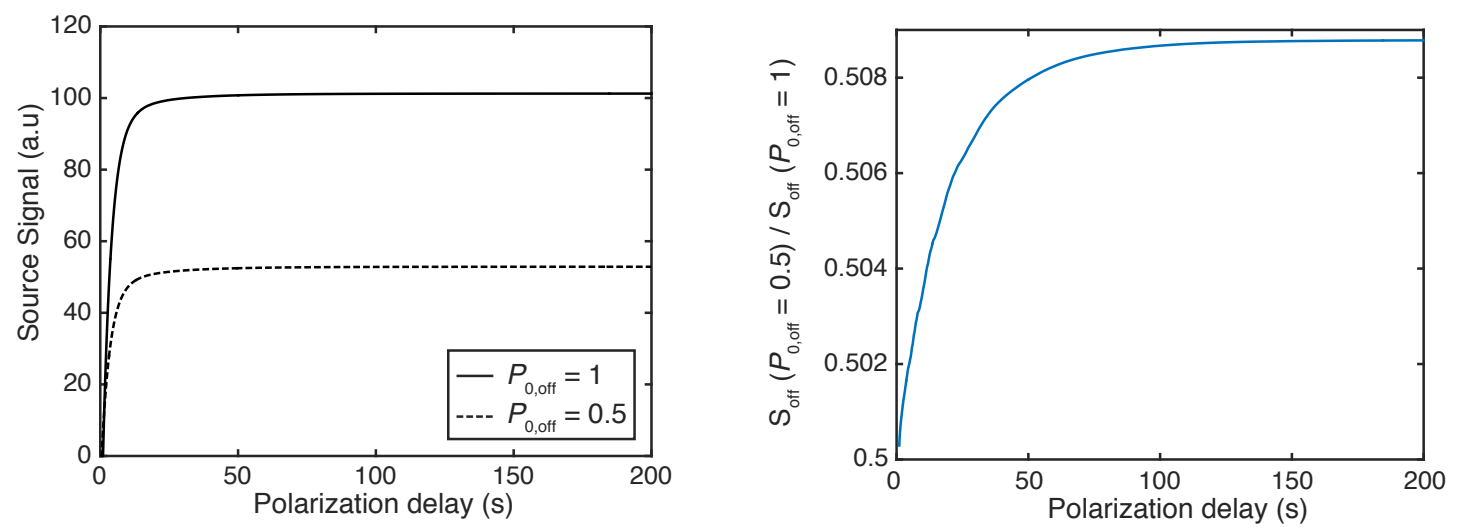

Figure S11. Left: Source signals without $\mu$ wave irradiation in a 3D system as a function of the polarization delay without depolarization $\left(P_{0, \text { off }}=1\right)$ and with depolarization $\left(P_{0, \text { off }}=0.5\right)$. Right: Ratio of the signals of the left figure. In the simulation, $\mathrm{L}=1 \mu \mathrm{m}, T_{1, \text { source }}=3 \mathrm{~s}, T_{1 \text {,target }}=30 \mathrm{~s}, \varepsilon_{0}=150, D_{\text {target }}=D_{\text {source }}$ $=1.10^{-3} \mu \mathrm{m}^{2} . \mathrm{s}^{-1}, P_{\mathrm{L}}=0.2 \mu \mathrm{m}, \theta_{\min }=1$, and the border was $2 \mathrm{~nm}$ thick.

Analogous observation is done for a system when the microwave irradiation is effective. We see that the ratio of the local equilibrium polarizations is quite close to the ratio of the signals. We assumed that both were equal for typical values of the relaxation times and diffusion coefficient. We note that if the relaxation time of the source and the target are in the same order of magnitude, this approximation will no longer be appropriate for a heterogeneous system. 

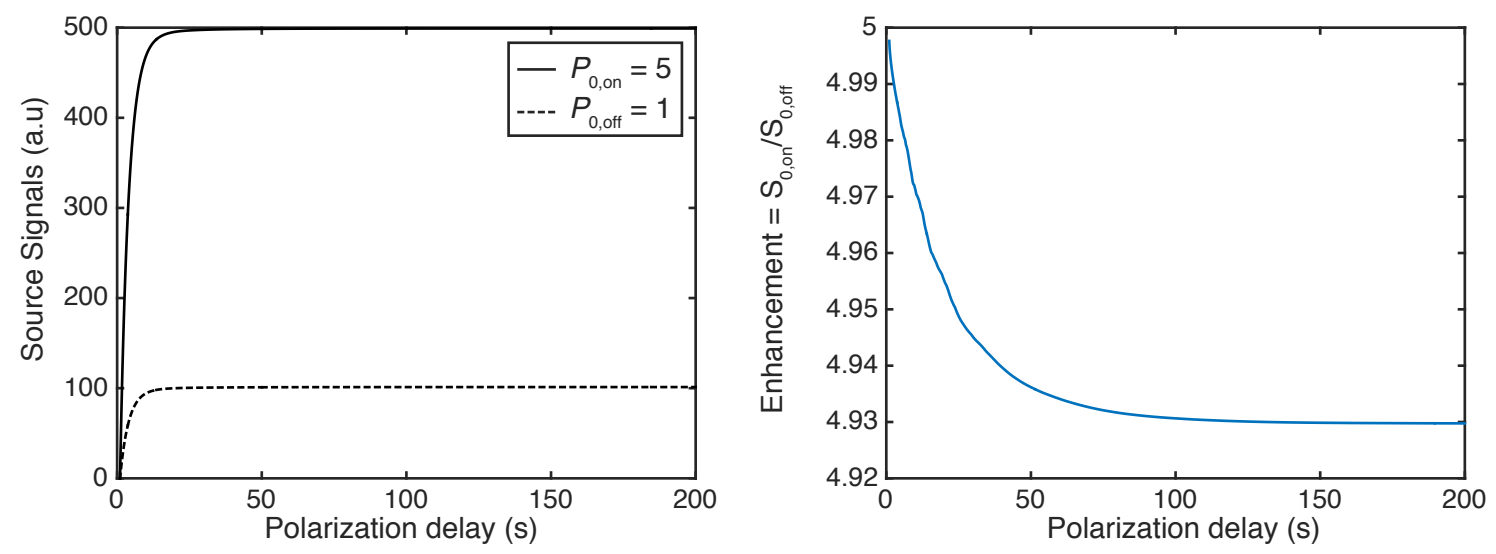

Figure S12. Left: Source signals with and without $\mu$ wave irradiation in a 3D system as a function of the polarization delay. Right: Ratio of the signals of the left figure (thus, the enhancement of the source as a function of the polarization delay). In the simulation, $\mathrm{L}=1 \mu \mathrm{m}, T_{1, \text { source }}=3 \mathrm{~s}, T_{1, \text { target }}=30 \mathrm{~s}, \varepsilon_{0}=5, D_{\text {target }}$ $=D_{\text {source }}=1.10^{-3} \mu \mathrm{m}^{2} \mathrm{~s}^{-1}, P_{\mathrm{L}}=0.2 \mu \mathrm{m}, \theta_{\min }=1$, and the border was $2 \mathrm{~nm}$ thick.

We see in Figure S12 that the source enhancement can be considered as constant, which is in agreement with what we observe with the TCE or glycerol enhancement in the main paper.

\section{$\beta$ factor of the stretched exponential fitting functions as a function of the length of the target:}
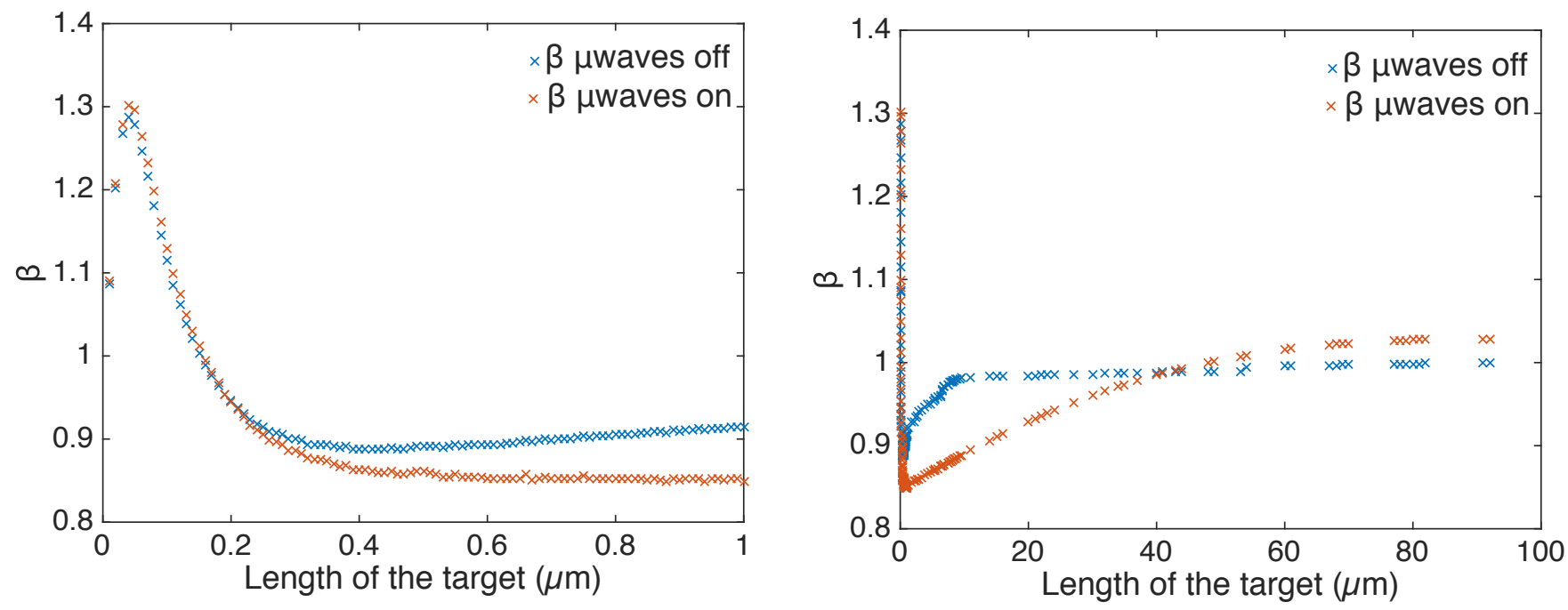

Figure S13. Values of $\beta$ of the stretched exponential fitting function as a function of the length of the target for a spherically symmetric system. All simulations parameters are given in the result section of the main text. 


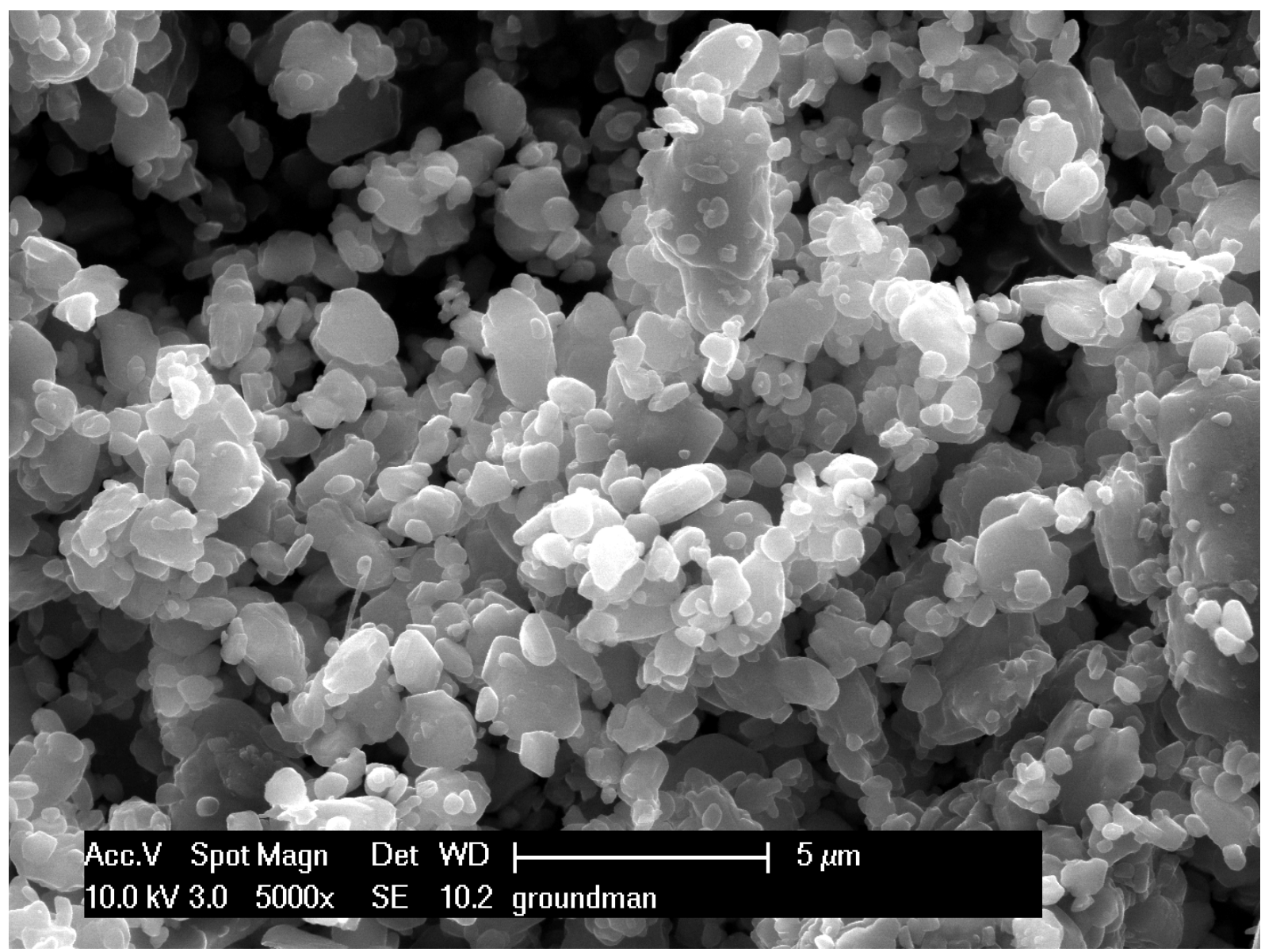

Figure S14. SEM image of the Histidine powder manually ground for 5 minutes in a mortar.

Size distribution of EC lengths for the aquacoat study
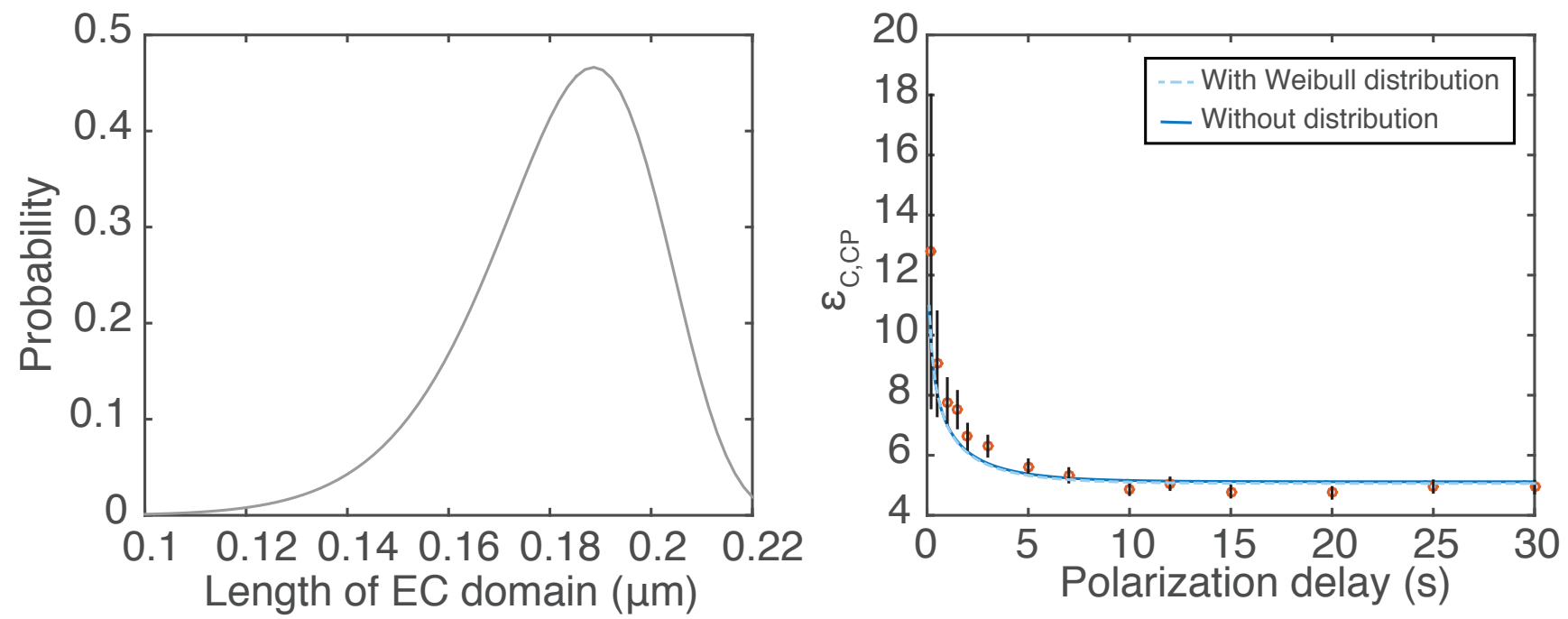

Figure S15. Left: Weibull distribution of EC lengths used for the simulation. Right: Comparison of the enhancements with and without a distribution of EC lengths. 
(a)
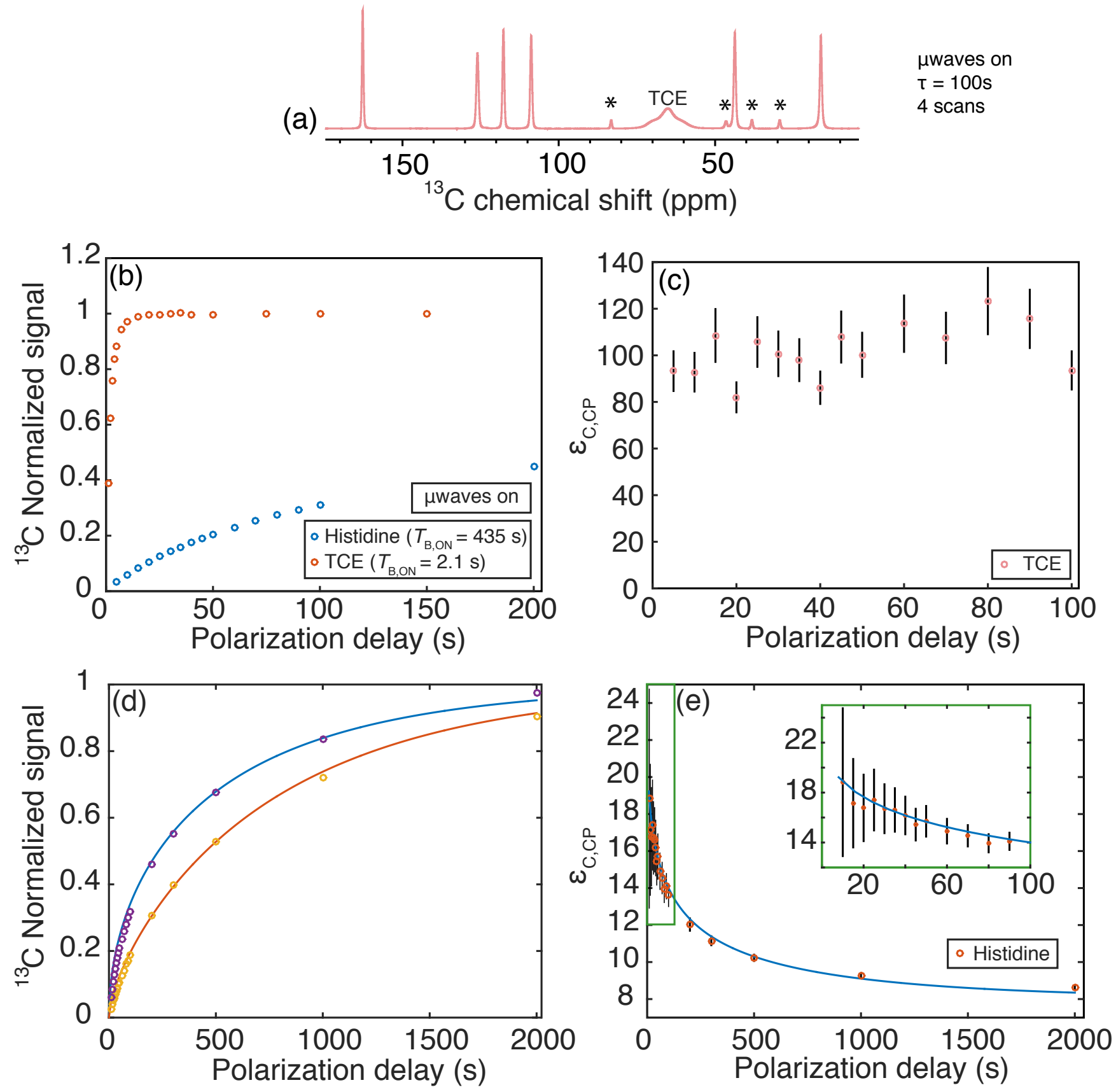

Figure S16. (a) ${ }^{13} \mathrm{C}$ CPMAS DNP enhanced solid-state NMR spectra obtained at $105 \mathrm{~K}, \mathrm{~B}_{0}=9.4 \mathrm{~T}$ and $8 \mathrm{kHz}$ MAS of microcrystalline histidine which was ground by hand for 5 minutes and impregnated with a solution of TCE containing TEKPol. (b) ${ }^{13} \mathrm{C}$ CPMAS normalized signal build-up of the TCE peaks with and without microwave irradiation. Normalization was performed using the value of the signals at steady from a stretched exponential fit. (c) ${ }^{13} \mathrm{C} \mathrm{CP}$ DNP enhancement of TCE peaks as a function of the polarization delay. (d) Build-up of histidine peaks with and without microwave irradiation as a function of the polarization delay. (d) ${ }^{13} \mathrm{C}$ CP DNP enhancement of histidine peaks as a function of the polarization delay. The histidine build-up points correspond to the average of the integrated areas of all the histidine ${ }^{13} \mathrm{C}$ peaks. Error bars were calculated by propagation of error using the noise levels of the spectra acquired with and without microwave irradiation as the standard deviation. In Figures (d) and (e) 
numerical simulations are overlapped for particle with spherical symmetry with: $L=7 \mu \mathrm{m}, T_{\mathrm{B}, \text { source }}=5$ $\mathrm{ms}, T_{1, \text { target }}=1038 \mathrm{~s}, D_{\text {target }}=1,2.10^{-4} \mu \mathrm{m}^{2} \mathrm{~s}^{-1}, D_{\text {source }}=3.10^{-5} \mu \mathrm{m}^{2} \mathrm{~s}^{-1}, P_{\mathrm{L}}=1 \mu \mathrm{m}$, and $\varepsilon_{\text {Depo }}=0.5$.

Why does the enhancement increase at early times for thick borders?

$$
\frac{\mathrm{d} \varepsilon}{\mathrm{dt}}>0 \Leftrightarrow \frac{\mathrm{d}\left(\frac{P_{O N}}{P_{O F F}}\right)}{\mathrm{dt}}>0 \Leftrightarrow \frac{\mathrm{d} P_{O N}}{\mathrm{dt}} P_{O F F}-\frac{\mathrm{d} P_{O F F}}{\mathrm{dt}} P_{O N}>0 \Leftrightarrow \frac{\frac{\mathbf{d} \boldsymbol{P}_{O N}}{\mathbf{d t}}(\boldsymbol{t})}{\boldsymbol{\varepsilon}(\mathbf{t})}>\frac{\mathbf{d} \boldsymbol{P}_{\text {OFF }}(\boldsymbol{t})}{\mathbf{d t}}
$$

The above shows that asserting that the enhancement increases with polarization time means that the polarization with $\mu$ waves on, scaled by the enhancement, builds up faster than the polarization without $\mu$ waves. This last condition has to be satisfied for any time during which the enhancement is increasing.

To verify this condition for the thick border case, we plot in Figure S17 the polarizations with and without $\mu$ waves in the target for a region next to the border for two early time points ( 1 and 2 seconds). We then scale the $\mu$ waves on polarizations by the average of the enhancement between 1 and 2 seconds (the enhancement is 42 at $1 \mathrm{~s}$ and 58 at $2 \mathrm{~s}$ so we choose to scale the polarization by 50).

In this way we see that the polarization has increased relatively more in presence of $\mu$ waves than without $\mu$ waves (see Figure S17a). This thus satisfies the condition above for the thick border case. In the thin border case, the polarization increases more without $\mu$ waves than with $\mu$ waves, and the condition is not fulfilled: the enhancement decreases at early times.

The fact that the target polarization increases more rapidly with than without $\mu$ waves in the thick border case comes from the fact that a small region of the target also contains sources. Since the border is thick, the hyperbolic secant functions are broad enough to overlap significantly with the target region. When polarization builds up in this target region, relatively more polarization diffuses out of the region by spin diffusion in the $\mu$ waves off than $\mu$ waves on case (since longitudinal relaxation reduces diffusion in the $\mu$ waves on case, and enhances diffusion in the $\mu$ waves off case). Consequently, at a given time, a larger part of the polarization is present outside this region in the $\mu$ waves off case than in the $\mu$ waves on case. Thus, the overall build-up is slower without than with microwaves.
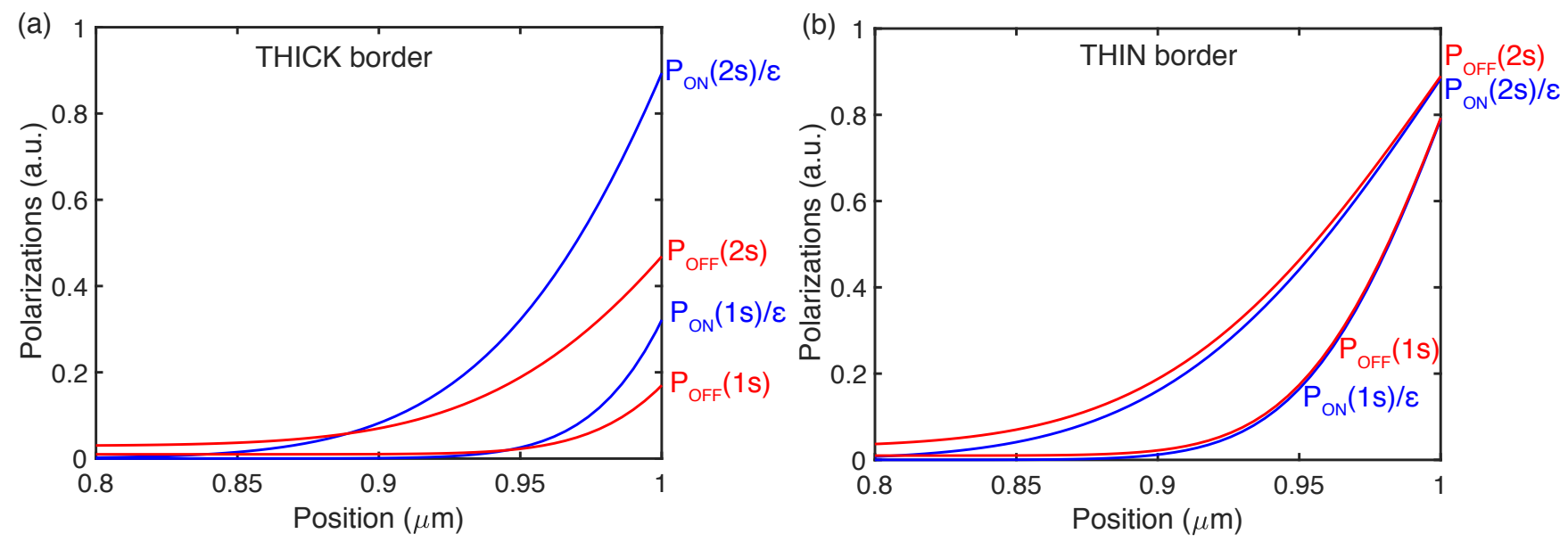

Figure S17. Simulated polarization curves as a function of the position in the target, in the volume next to the border with the source (the border is at $1 \mu \mathrm{m}$ on this scale). The plotted polarizations correspond to the polarization at 1 and 2 seconds after saturation in absence of microwaves $\left(P_{\mathrm{OFF}}\right.$, red) and in presence of microwaves $\left(P_{\mathrm{ON}}\right.$, blue) in the (a) thick border and (b) thin border cases. The polarizations with microwaves were scaled down by the enhancement since the actual hyperpolarization value is much higher than 1, since the DNP effect is occurring. The system was considered to have a spherical symmetry, $T_{1, \text { source }}=100 \mathrm{~ms}, T_{1 \text {,target }}=100 \mathrm{~s}, \mathrm{D}_{\text {source }}=\mathrm{D}_{\text {target }}=10^{-3} \mu \mathrm{m}^{2} \mathrm{~s}^{-1}, P_{\mathrm{L}}=1 \mu \mathrm{m}, P_{0, \mathrm{ON}}=100$ and $\varepsilon_{\text {Depo }}=1$. 


\section{Definition of Characteristic diffusion length and characteristic diffusion time.}

The Fick diffusion equation for the spin-diffusion process is defined as:

$$
\frac{\partial P(x, t)}{\partial t}=D(x) \cdot \Delta P-\frac{P(x, t)-P_{0}(x)}{T_{1}(x)}
$$

where $x$ is the position vector distance from the border between source and target, $t$ is the time $(0<t<$ $\infty), P$ is the instantaneous polarization, $P_{0}$ is the local equilibrium polarization, $D$ is the diffusion rate at position $x, T_{1}$ is the longitudinal relaxation time at position $x$, and $\Delta P$ is the Laplacian.

The detailed expression of the Laplacian depends on the geometry of the system to be investigated. In the main text we are analysis three different geometries corresponding to linear, cylindrical and spherical symmetry. In all of these descriptions only one of the three coordinates is relevant since, for example in a system with spherical symmetry, the polarization function $P$ does not explicitly depend in the angular coordinates $(\theta, \phi)$, but only in the radial coordinate.

In these cases, the Fick equation can be written as equation (2):

$$
\frac{\partial P(r, t)}{\partial t}=D(r) \cdot\left[r^{1-m} \frac{\partial}{\partial r}\left(r^{m-1} \frac{\partial P(r, t)}{\partial r}\right)\right]-\frac{P(r, t)-P_{0}(r)}{T_{1}(r)}
$$

where $r$ is the relevant "radial" coordinate and $m$ is parameter that describes the symmetry of the system. For the spherical symmetry we have $m=3$, in cylindrical coordinates $m=2$ and for the planar symmetry $m=1$. In the latter case the Fick equation has the same expression as the mono dimensional linear diffusion.

If we consider a domain (source or target) sufficiently homogenous to reasonably assume that the diffusion constant $D$ and the relaxation time $T_{1}$ are uniform constant with the domain, it becomes convenient to describe eq. (2) introducing two modified variables: the characteristic diffusion length $\rho$,

$$
\rho=\frac{r}{\sqrt{D T_{1}}}
$$

and the characteristic diffusion time $\tau$,

$$
\tau=\frac{t}{T_{1}}
$$

In these variables equation (2) becomes the following characteristic equation:

$$
\frac{\partial P(\rho, \tau)}{\partial \tau}=\rho^{1-m} \frac{\partial}{\partial \rho}\left(\rho^{m-1} \frac{\partial P(\rho, \tau)}{\partial \rho}\right)-\left(P(\rho, \tau)-P_{0}(\rho)\right)
$$

which does not depend anymore on the $D$ and $T_{1}$ constants, since these are implicitly included in the characteristic variables. Whatever will be the specific solution of eq. (4), it is thus possible to find a general solution, independent from $D$ and $T_{1}$, and to analyze the behavior in terms of the characteristic length and time. In any investigated systems we expect to have different behaviors if $\rho, \tau>>1$ or $\rho, \tau$ $<<1$ :

$$
\rho \ll 1, \quad r \ll \sqrt{D T_{1}}
$$

and

$$
\rho \gg 1, \quad r \gg \sqrt{D T_{1}}
$$


Equations (5) can be extended for a system where $D$ and $T_{1}$ are not a constant but a function of the position defining characteristic diffusion length and time on the bases of the average values inside the domain:

$$
\rho \ll 1, \quad r \ll \sqrt{\langle D\rangle\left\langle T_{1}\right\rangle}
$$

and

$$
\rho \gg 1, \quad r \gg \sqrt{\langle D\rangle\left\langle T_{1}\right\rangle}
$$

which is the definition reported in the main text.

\section{Solution for the linear case}

As an example of the meaning of the characteristic length we report here the analytic solution for the linear diffusion. The equation for linear diffusion can be derived from equation (2) using $m=1$, and we assume $D, T_{1}$, as well as the equilibrium polarization $P_{0}$, constant inside the target volume:

$$
\frac{\partial P(r, t)}{\partial t}=D \cdot \frac{\partial^{2} P(r, t)}{\partial r^{2}}-\frac{P(r, t)-P_{0}}{T_{1}}
$$

If we call:

$$
P(r, t)-P_{0}=P^{\prime}(r, t)
$$

we have:

$$
\begin{aligned}
& \frac{\partial P^{\prime}(r, t)}{\partial t}=\frac{\partial P(r, t)}{\partial t} \\
& \frac{\partial^{2} P^{\prime}(r, t)}{\partial r^{2}}=\frac{\partial^{2} P(r, t)}{\partial r^{2}}
\end{aligned}
$$

with these conditions, the equation (7) become:

$$
\frac{\partial P^{\prime}(r, t)}{\partial t}=D \cdot \frac{\partial^{2} P \prime(r, t)}{\partial r^{2}}-\frac{P \prime(r, t)}{T_{1}}
$$

and for the steady state (homogenous equation), equation (10) becomes:

$$
D \cdot \frac{\partial^{2} P^{\prime}(r, t)}{\partial r^{2}}-\frac{P^{\prime}(r, t)}{T_{1}}=0
$$

It can be seen that the general solution for equation (11) is:

$$
P^{\prime}(r, \infty)=A e^{\frac{r}{\sqrt{D T_{1}}}}+B e^{-\frac{r}{\sqrt{D T_{1}}}}=(A+B) \cosh \left(\frac{r}{\sqrt{D T_{1}}}\right)+(A-B) \sinh \left(\frac{r}{\sqrt{D T_{1}}}\right)
$$

and the steady-state polarization $P(r, \infty)$ is:

$$
P(r, \infty)=A e^{\frac{r}{\sqrt{D T_{1}}}}+B e^{-\frac{r}{\sqrt{D T_{1}}}}+P_{0}=(A+B) \cosh \left(\frac{r}{\sqrt{D T_{1}}}\right)+(A-B) \sinh \left(\frac{r}{\sqrt{D T_{1}}}\right)+P_{0}
$$

where $A$ and $B$ constants are determined from the boundary conditions.

We can see that the radial dependence $r$ is scaled by the term $\sqrt{D T_{1}}$ as expected form the characteristic diffusion length. Equation (12) is in fact a function of the characteristic length $\rho$ (eq. 3a), which make possible to generalize the behavior for samples with different $D$ and $T_{1}$. 


\section{Analytic solution for the $\varepsilon_{\text {target, } \infty}$ for linear and spherical symmetry.}

\section{Linear symmetry}

The Fick equation for the linear symmetry in the steady state limit assuming $D$ and $T_{1}$ constant, and the equilibrium polarization $P_{0}$ being equal to 1 inside the target volume, is:

$$
D \cdot \frac{\partial^{2} P(r, t)}{\partial r^{2}}-\frac{P(r, t)-1}{T_{1}}=0
$$

It is convenient to set the center of the target domain as the origin of the $r$ coordinates (Figure S18a), in this condition we can find that solution of the Fick equation is:

$$
P(r, \infty)=\frac{P_{\text {source }}-1}{\cosh \left(\frac{L}{2 \sqrt{D T_{1}}}\right)} \cosh \left(\frac{r}{\sqrt{D T_{1}}}\right)+1
$$

where $P_{\text {source }}$ is the source polarization, $L$ is the total length of the target. As boundary conditions we

assumed that at the border between target and source $\left(r= \pm \frac{L}{2}\right)$ the steady state polarization is $P\left( \pm \frac{L}{2}, \infty\right)=P_{\text {source }}$.

We also assume that, in absence of microwave $P_{\text {source, } \mathrm{OFF}}=1$, which means that don't take into account depolarization phenomena. As a consequence, $P_{\text {source, } \mathrm{ON}}=\varepsilon_{\text {source }}$. In these conditions, by integrating the equation (15) along the target length, we obtain the enhancement $\varepsilon_{\text {target,o }}$ as:

$$
\varepsilon_{\text {target }, \infty}=1+\left(\varepsilon_{\text {source }}-1\right) \frac{2 \sqrt{D T_{1}}}{L} \tanh \left(\frac{L}{2 \sqrt{D T_{1}}}\right)
$$

This is the general solution of Fick's equation in a linear symmetry.

\section{Cylindrical symmetry}

The Fick equation for the cylindrical symmetry in the steady state limit and assuming $D, T_{1}$, and the equilibrium polarization $P_{0}$, being equal to 1 inside the target domain volume is:

$$
D \cdot\left(\frac{\partial^{2} P(r, t)}{\partial r^{2}}+\frac{1}{r} \frac{\partial P(r, t)}{\partial r}\right)-\frac{P(r, t)-1}{T_{1}}=0
$$

It is convenient to set the center of the cylindrical target domain as the origin of the $r$ coordinates (Figure S18a), in this condition we can find that solution of the Fick equation is:

$$
P(r, \infty)=\frac{\left(P_{\text {source }}-P_{0}\right)}{I_{0}\left(\frac{L}{\sqrt{D T_{1}}}\right)} I_{0}\left(\frac{r}{\sqrt{D T_{1}}}\right)+P_{0}
$$

where $P_{\text {source }}$ is the source polarization, $L$ is the length of the target cylinder and $I_{0}(x)$ is the modified Bessel function of the zero order. As boundary conditions we assume that at the border between target and source $(r=L)$ the polarization is $P(L, \infty)=P_{\text {source }}$.

We also assume that, in absence of microwave $P_{\text {source,OFF }}=1$, which means that don't take into account depolarization phenomena. As a consequence, $P_{\text {source, } \mathrm{ON}}=\varepsilon_{\text {source }}$. In these conditions, by integrating the equation (18) along the target length, we obtain the enhancement $\varepsilon_{\text {target,o }}$ as: 


$$
\varepsilon_{\text {target }, \infty}=1+\left(\varepsilon_{\text {source }}-1\right) \frac{2 \sqrt{D T_{1}}}{L}\left[\frac{I_{1}\left(\frac{L}{\sqrt{D T_{1}}}\right)}{I_{0}\left(\frac{L}{\sqrt{D T_{1}}}\right)}\right]
$$

where $I_{0}(x)$ and $I_{1}(x)$ are the modified Bessel function of the zero and first order, respectively. This is the general solution of Fick's equation in a cylindrical symmetry.

\section{Spherical symmetry}

The Fick equation for the spherical symmetry in the steady state limit assuming $D$ and $T_{1}$ constant, and the equilibrium polarization $P_{0}$ being equal to 1 inside the target volume, is:

$$
D \cdot\left(\frac{\partial^{2} P(r, t)}{\partial r^{2}}+\frac{2}{r} \frac{\partial P(r, t)}{\partial r}\right)-\frac{P(r, t)-1}{T_{1}}=0
$$

It is convenient to set the center of the target domain as the origin of the $r$ coordinates (Figure S18b), in this condition we can find that solution of the Fick equation is:

$$
P(r, \infty)=\frac{P_{\text {source }}-1}{\sinh \left(\frac{L}{\sqrt{D T_{1}}}\right)} \frac{L}{\sqrt{D T_{1}}} \frac{\sinh \left(\frac{r}{\sqrt{D T_{1}}}\right)}{r}+1
$$

where $P_{\text {source }}$ is the source polarization, $\mathrm{L}$ is the total radius of the target sphere. As boundary conditions we assume that at the border between target and source $(r=L)$ the polarization is $P(L, \infty)=$ $P_{\text {source }}$ which means a thin border and a high diffusion rate inside the source domain.

We also assume that, in absence of microwave $P_{\text {source, } \mathrm{OFF}}=1$, which means that don't take into account depolarization phenomena. As a consequence, $P_{\text {source, } \mathrm{ON}}=\varepsilon_{\text {source }}$. In these conditions, by integrating the equation (21) along the target volume, we obtain the enhancement $\varepsilon_{\text {target,o }}$ as:

$$
\varepsilon_{\text {target }, \infty}=1+\left(\varepsilon_{\text {source }}-1\right) \frac{3 \sqrt{D T_{1}}}{L}\left[\operatorname{coth}\left(\frac{L}{\sqrt{D T_{1}}}\right)-\frac{\sqrt{D T_{1}}}{L}\right]
$$

This is the general solution of Fick's equation in a spherical symmetry.

\section{Enhancement equations}

The comparison of the $\varepsilon_{\text {target, } \infty}$ for different symmetries is more appropriate if we compare targets of radius $L$ in the spherical and cylindrical symmetries with targets of length $2 L$ for the linear symmetry. The equations for $\varepsilon_{\text {target, } \infty}$ become:

\section{Linear symmetry}

$$
\varepsilon_{\text {target }, \infty}=1+\left(\varepsilon_{\text {source }}-1\right) \frac{\sqrt{D T_{1}}}{L} \tanh \left(\frac{L}{\sqrt{D T_{1}}}\right)
$$

Cylindrical symmetry

$$
\varepsilon_{\text {target }, \infty}=1+\left(\varepsilon_{\text {source }}-1\right) \frac{2 \sqrt{D T_{1}}}{L}\left[\frac{I_{1}\left(\frac{L}{\sqrt{D T_{1}}}\right)}{I_{0}\left(\frac{L}{\sqrt{D T_{1}}}\right)}\right]
$$

Spherical symmetry 


$$
\varepsilon_{\text {target }, \infty}=1+\left(\varepsilon_{\text {source }}-1\right) \frac{3 \sqrt{D T_{1}}}{L}\left[\operatorname{coth}\left(\frac{L}{\sqrt{D T_{1}}}\right)-\frac{\sqrt{D T_{1}}}{L}\right]
$$

We can plot the enhancement as a function of $L$ for $D=10^{-3} \mu \mathrm{m}^{2} \mathrm{~s}^{-1}$ and $T_{1}=40 \mathrm{~s}$, we obtain:

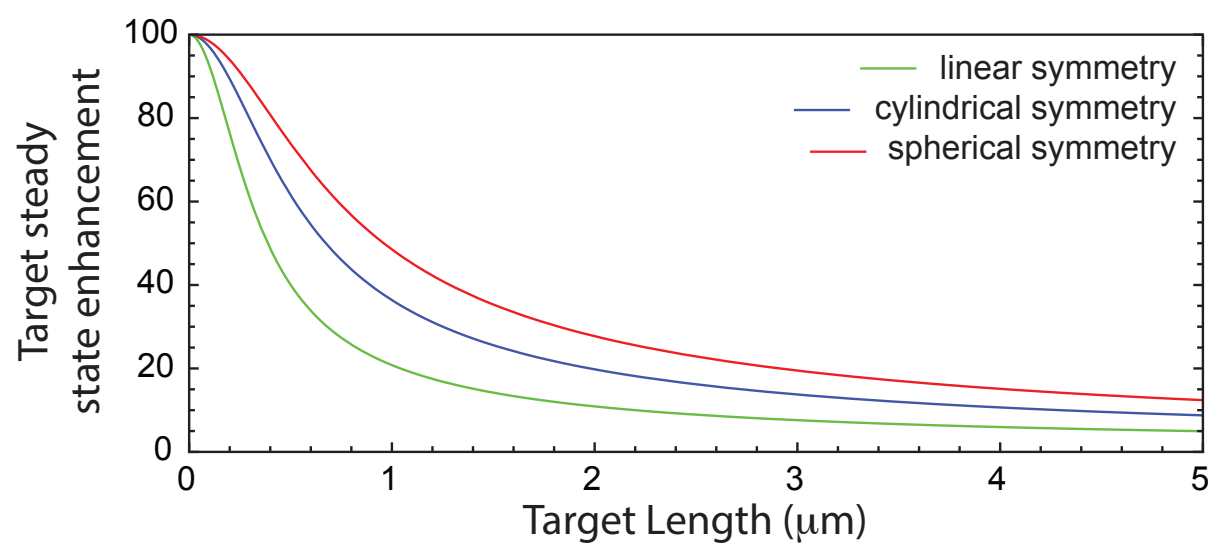

where we notice that going towards a spherical symmetry, enhancement increases. Mathematically, this is explained by the increasing number $m$ in the factor $\frac{m \sqrt{D T_{1}}}{L}$ in the middle of the previous equations. The physical reason for this different trend is because the different symmetries have different ratio between the polarizing surface (S) in contact with the source, and the target volume to be polarized (V). This ratio is $\frac{S}{V}=\frac{m}{L}$, with $\mathrm{m}=1,2,3$, for linear, cylindrical and spherical case, respectively, and $\mathrm{L}$ is the size. This means that for the same size, the spherical case has more polarizing surface for unit of volume, which explains the increasing hyperpolarization and enhancement. 


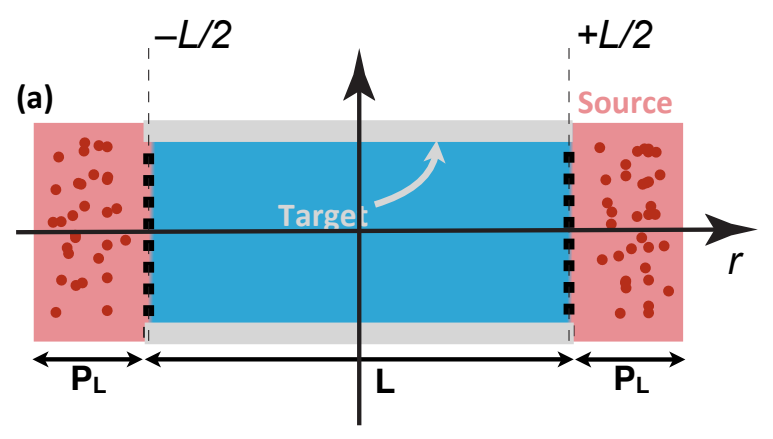

(b)

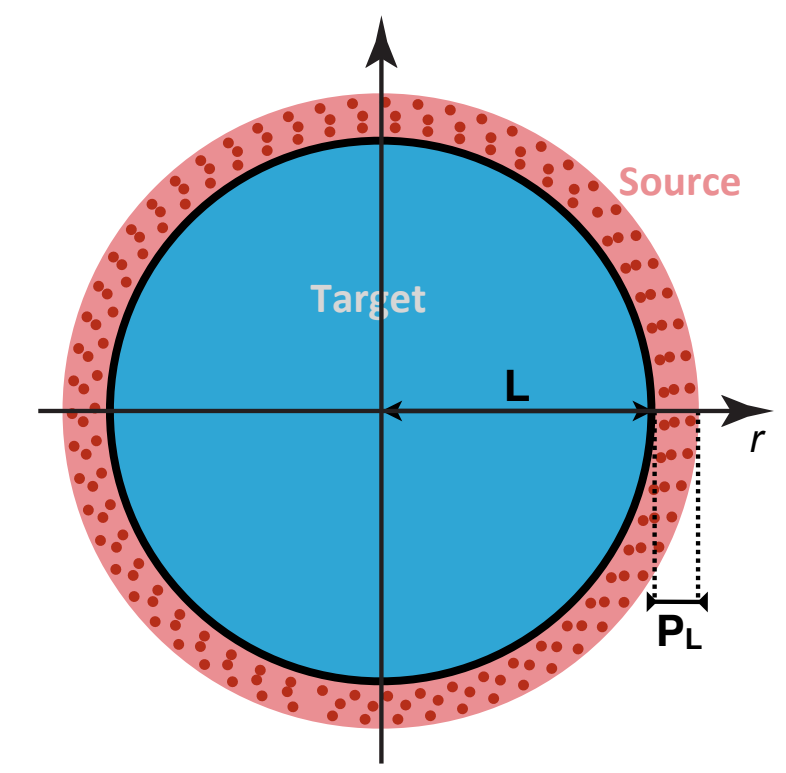

Figure S18. Coordinates Frame reference for equations used in the linear and spherical symmetry description. 

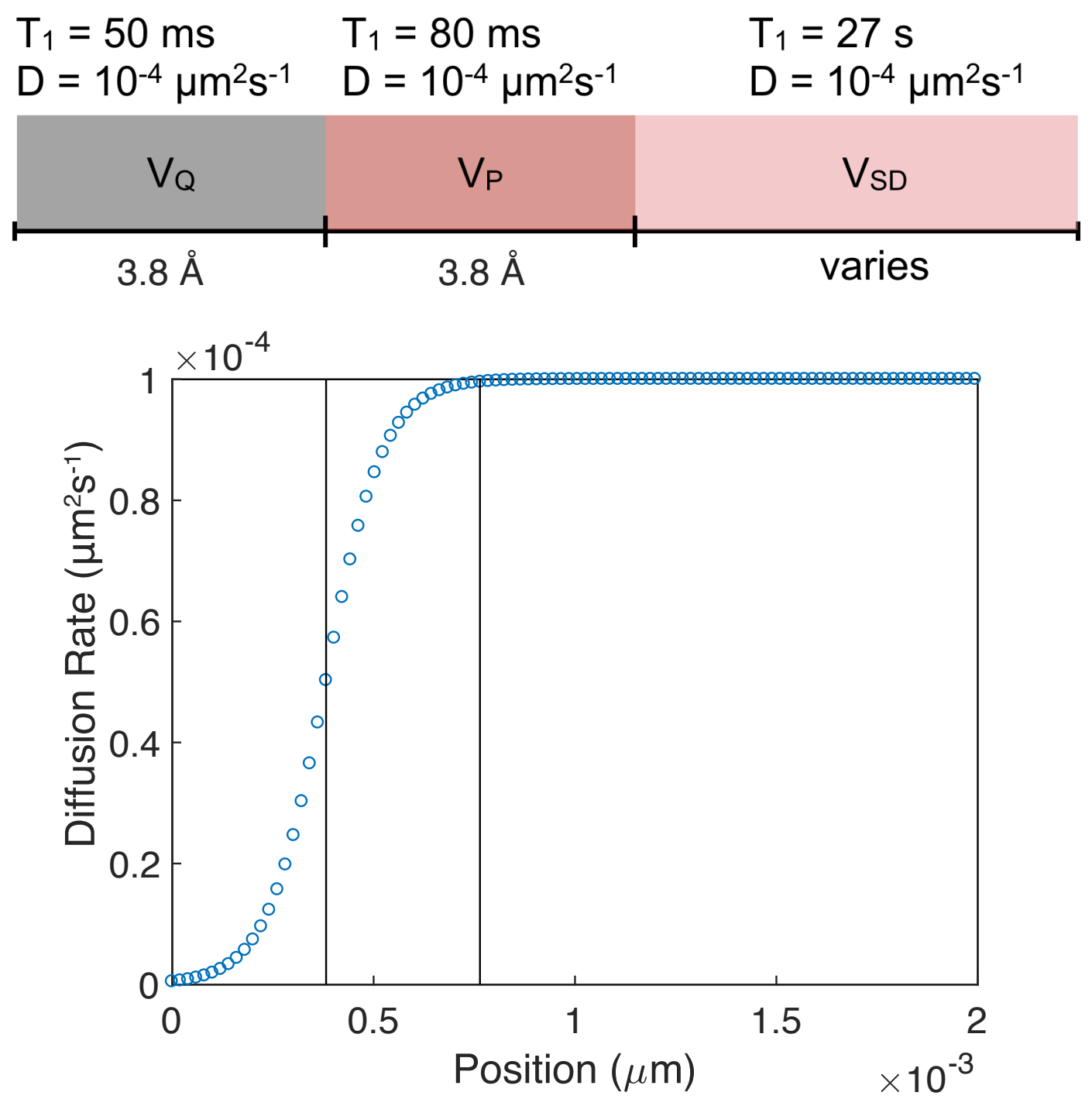

Figure S19. Simulated diffusion rate profile as a function of the position, close to the electron location.

The profile shown in Figure S19 is the one used for the simulations of the main text. Another model where the diffusion rate was kept constant at $10^{-3} \mu \mathrm{m}^{2} \cdot \mathrm{s}^{-1}$ as a function of the position, and gave slightly worse fit, with only slightly different $T_{1} \mathrm{~s}$, as shown in the following. 


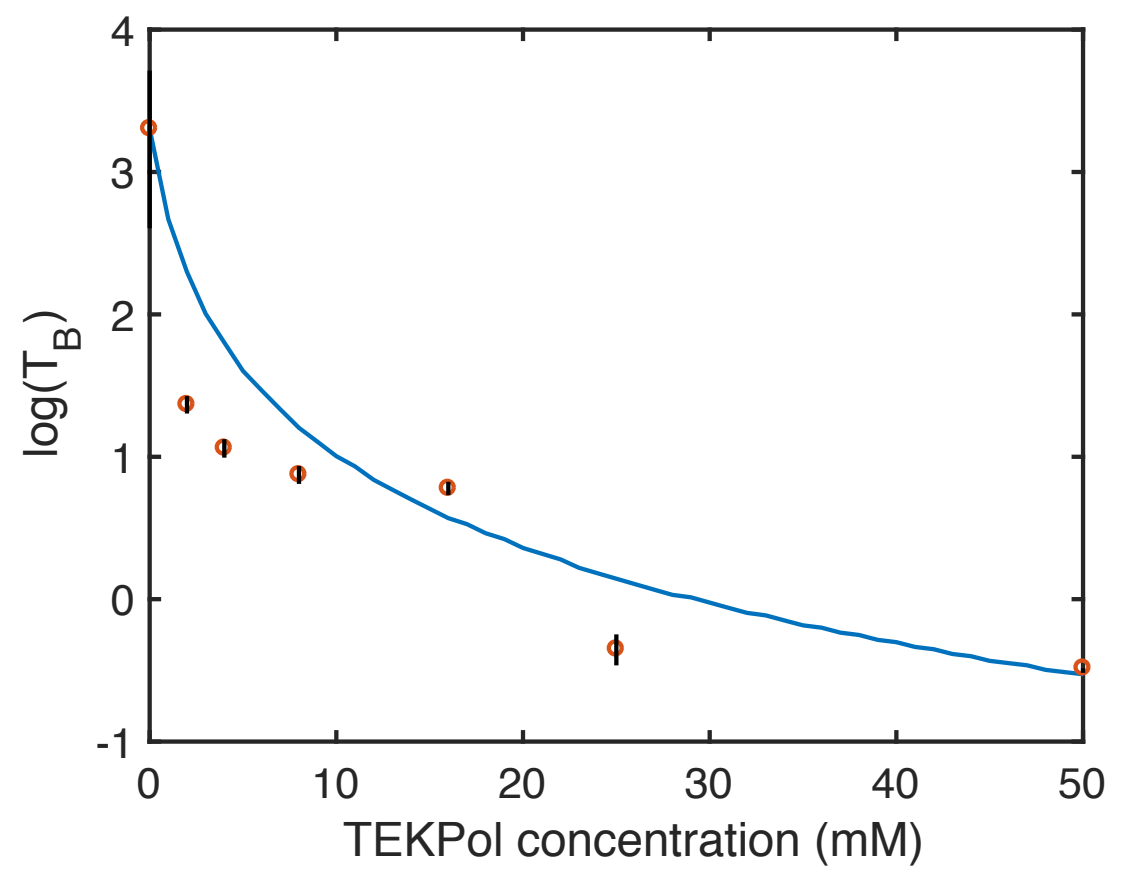

Figure S20. Experimental ${ }^{1} \mathrm{H}$ build-up time $T_{\mathrm{B}}$ as a function of the concentration of TEKPol in a frozen solution of TCE at $105 \mathrm{~K}$, with $8 \mathrm{kHz}$ sample spinning recorded on a $500 \mathrm{MHz}$ spectrometer, using saturation recovery and determined using a mono exponential fit. All samples were degassed using 5 inserteject cycles prior to measurement to minimize additional paramagnetic relaxation due to dissolved oxygen. ${ }^{3}$ The solid blue line is the result of a numerical simulation for a source and target according to Figure 2a of the main text for which at each concentration the build-up was simulated and then fit to a mono exponential. In the simulations, the system was assumed to be three-dimensional. $T_{1, \mathrm{VSD}}$ was measured to be $27 \mathrm{~s}$ (in pure TCE). The size of the volumes $V_{\mathrm{Q}}$ and $V_{\mathrm{P}}$ were determined by fitting to be both $3.8 \pm 0.1 \AA$. $T_{1, \mathrm{VQ}}$ and $T_{1, \mathrm{VP}}$ were determined by fitting to be $50 \pm 10 \mathrm{~ms}$ and $30 \pm 10 \mathrm{~ms}$ respectively.

\section{References}

(1) Maricq, M. M.; Waugh, J. S. NMR in rotating solids J. Chem. Phys. 1979, 70, 3300-3316.

(2) Thurber, K. R.; Tycko, R. Perturbation of nuclear spin polarizations in solid state NMR of nitroxidedoped samples by magic-angle spinning without microwaves J. Chem. Phys. 2014, 140.

(3) Kubicki, D. J.; Rossini, A. J.; Purea, A.; Zagdoun, A.; Ouari, O.; Tordo, P.; Engelke, F.; Lesage, A.; Emsley, L. Amplifying Dynamic Nuclear Polarization of Frozen Solutions by Incorporating Dielectric Particles J. Am. Chem. Soc. 2014, 136, 15711-15718. 